\title{
A PRELIMINARY PROPOSAL FOR QUALITY CONTROL ASSESSMENT AND HARMONIZATION OF LEUKOCYTES MORPHOLOGY-STRUCTURAL PARAMETERS (CELL POPULATION DATA PARAMETERS)
}

\author{
PRELIMINARNI PREDLOG ZA PROCENU KONTROLE KVALITETA I HARMONIZACIJU \\ MORFOLOŠKO-STRUKTURNIH PARAMETARA LEUKOCITA \\ (PARAMETARA PODATAKA O ĆELIJSKOJ POPULACIJI)
}

\author{
Michela Seghezzi ${ }^{1}$, Sabrina Buoro ${ }^{1}$, Giulia Previtali ${ }^{1}$, Valentina Moioli ${ }^{1}$, Barbara Manenti ${ }^{1}$, \\ Ramon Simon-Lopez ${ }^{2}$, Cosimo Ottomano ${ }^{3}$, Giuseppe Lippi ${ }^{4}$ \\ ${ }^{1}$ Clinical Chemistry Laboratory, Hospital Papa Giovanni XXIII, Bergamo, Italy \\ ${ }^{2}$ Sysmex Corporation, Japan \\ ${ }^{3}$ Synlab, Castenedolo, Italy \\ ${ }^{4}$ Section of Clinical Biochemistry, University of Verona, Verona, Italy
}

\begin{abstract}
Summary
Background: The cell population data (CPD) measured by Sysmex XN-9000 can be used for screening many hematological and non-hematological disorders. Since little information is available on harmonization of CPD among different instrumentation and clinical laboratories, this study aimed at assessing the current degree of CPD harmonization between separate Sysmex $\mathrm{XN}$ modules allocated to the same laboratory.

Methods: A total number of 78291 data were used for verification of within-run imprecision, analyzers harmonization, reference ranges and assessment of blood sample stability of CPD parameters, including results of daily quality control testing and those generated in samples collected from blood donors and healthy volunteers.

Results: Within-run imprecision of CPD parameters ranged between 0.4 and $14.1 \%$. Good agreement was found among five different $\mathrm{XN}$-modules, especially when values were adjusted after calculation of instrument-specific alignment factors. The bias of all parameters remained always
\end{abstract}

\section{Address for correspondence:}

Sabrina Buoro

Clinical Chemistry Laboratory - Papa Giovanni XXIII Hospital, Piazza OMS, 1 - 24127 Bergamo, Italy

Phone: (+039) 0352674550

Fax: (+039) 0352674939;

e-mail: sbuoro@asst-pg23.it

\section{Kratak sadržaj}

Uvod: Podaci o ćelijskoj populaciji (cell population data, CPD) mereni pomoću uređaja Sysmex XN-9000 mogu se koristiti za skrining mnogih hematoloških i nehematoloških poremećaja. Pošto je dostupno malo informacija o usklađivanju CPD između različitih instrumenata i kliničkih laboratorija, ova studija imala je za cilj procenu trenutnog stepena harmonizacije CPD između različitih Sysmex XN modula raspoloživih u istoj laboratoriji.

Metode: Ukupno 78291 podataka upotrebljeno je za verifikovanje nepreciznosti unutar serije, harmonizacije analizatora, referentnih opsega i pocenu stabilnosti uzoraka krvi za CPD parametre, uključujući rezultate svakodnevnog testiranja kontrole kvaliteta i one generisane u uzorcima sakupljenim od davalaca krvi i zdravih dobrovoljaca.

Rezultati: Nepreciznost unutar serije za CPD parametre kretala se između 0,4 i 14,1\%. Pronađeno je dobro slaganje između pet različitih $\mathrm{XN}$ modula, naročito pošto su vrednosti prilagođene posle računanja faktora poravnanja specifičnih za instrument. Odstupanje za sve parametre ostajalo je uvek

List of abbreviations: $\mathrm{B}_{\mathrm{ALG}}$, alignment bias based on intra-individual biological variation; $B_{A P S}$, analytical performance specification for bias; $\mathrm{CV}_{\mathrm{APS}}$, analytical performance specification for imprecision; $\mathrm{CV}_{\text {l, }}$, within-subject biological variation; $\mathrm{Cl}$, confidence interval; $\mathrm{CPD}$, cell population data; FSC, forward scatter; HA, hematological analyzers; HFLC, high fluorescence lymphocytes cell; IAF, instrumental alignment factor; LY, lymphocytes; LY-WX, lymphocyte complexity and width of dispersion of the events measured; LY-WY, lymphocyte fluorescence intensity and the width of dispersion; LY-WZ, lymphocyte cell size and the width of dispersion; LY-X, lymphocyte cell complexity; 
lower than the reference change values in samples stored for up to 8 hours, regardless of storage temperature.

Conclusions: The imprecision of CPD parameters was acceptable, except for those reflecting the dispersion of cellular clusters. Due to the lack of reference control materials, we showed that the use of data generated on a large number of normal routine samples (i.e., a Moving Average population) may be a reliable approach for testing analyzers harmonization. Nevertheless, availability of both calibration and quality control materials for these parameters is highly advisable in the future. We finally showed that whole blood samples may be stable for up to 2-4 hours for most CPD parameters.

Keywords: cell population data, quality control, withinrun imprecision, reference range, stability

\section{Introduction}

The development of innovative technologies and analytical principles in flow cytometry has been accompanied by commercialization of a new generation of hematological analyzers (HA) capable of generating both quantitative (i.e., cell counts and cellular indices) and qualitative (e.g., morphological flags, scattergrams) information, which also have a high degree of analytical efficiency for identifying many cellular abnormalities (1-3). The cell population data (CPD) measured by $\mathrm{HA}$ reflect some morphological parameters of white blood cell (WBC) subpopulations, and can be used for screening many hematological and non-hematological disorders. The Sysmex XN9000 (Sysmex Kobe Japan) not only measures conventional hematologic parameters, but can also generate innovative WBC parameters such as high fluorescence lymphocytes cell (HFLC) and CPD. The analytical techniques are essentially based on fluorescence flow cytometry for WBC differential count, and on a combination of forward scatter (FSC), side scatter (SSC) and fluorescence intensity (SFL) for detecting different WBC populations, respectively. These three measurements, together with their relative distribution width (W), can then be combined for obtaining CPD of neutrophils (NE), lymphocytes (LY) and monocytes (MO) populations.

Recent studies showed that NE CPD parameters (i.e., NE-SSC, NE-SFL) may be useful for diagnosing myelodysplastic syndromes and sepsis, whilst NE-SFL and MO-WX may play a role in differential diagnosis of some acute leukemias (4-9). Some LY CPD parameters (i.e., LY-X, LY-Y and LY-Z) may then pro-

LY-Y, lymphocyte fluorescence intensity; LY-Z, lymphocyte cell size; $M A$, moving average; $M C V$, mean corpuscular volume; $M O$, monocytes; MO-X, monocyte cell complexity; MO-Y, monocyte fluorescence intensity; MO-Z, monocyte cell size; MO-WX, monocyte complexity and width of dispersion of the events measured; MO-WY, monocyte fluorescence intensity and the width of dispersion; MO-WZ, monocyte cell size and the width of dispersion; NE: neutrophils; NEFSC, neutrophil cell size; NE-SFL, neutrophil fluorescence intensity; ispod referentnih vrednosti promena u uzorcima skladištenim do 8 sati, bez obzira na temperaturu skladištenja.

Zaključak: Nepreciznost CPD parametara bila je prihvatljiva, osim za one koji su odražavali rasipanje ćelijskih skupova. Usled nedostatka referentnih kontrolnih materijala, pokazali smo da upotreba podataka generisanih iz velikog broja normalnih rutinskih uzoraka (npr. Moving Average populacija) mogu služiti kao pouzdan pristup za testiranje harmonizacije analizatora. Uprkos tome, u budućnosti bi mnogo pomogla dostupnost kako kalibracije tako i materijala za kontrolu kvaliteta za ove parametre. Najzad, pokazali smo da uzorci pune krvi mogu biti stabilni i do 2-4 sata za većinu CPD parametara.

Ključne reči: podaci o ćelijskoj populaciji, kontrola kvaliteta, nepreciznost unutar serije, referentni opseg, stabilnost

vide valuable information for screening lymphoproliferative diseases $(10,11)$. Nevertheless, little information is available on the analytical quality specification of CPD, and even less is known on the degree of harmonization of these measures among different instrumentation and clinical laboratories. This last aspect is especially important due to the worldwide reorganization of laboratory diagnostics, which increasingly encompasses the shipment of blood specimens from one laboratory to another, thus potentially jeopardizing sample quality (12). Therefore, the aim of this study was to assess the current degree of CPD harmonization between separate Sysmex $\mathrm{XN}$ modules installed in the same laboratory.

\section{Materials and Methods}

\section{Description of the $\mathrm{XN}$-module hematological analyzer}

The $\mathrm{XN}-9000$ is the largest core model belonging to the XN-Series, being composed by up to seven separate analytical modules. A typical combination entails three "green « analyzers allocated for CBC and DIFF profiles, two »blue« analyzers allocated for AppRET, App PLT-F and App WPC analysis, along with a single slide preparation unit (Autoslider SP-10; Sysmex Co., Kobe, Japan) integrated with a slide processing system (DI60; Sysmex Co., Kobe, Japan). The analysis of all samples included in this study was carried out according to manufacturer's instructions. The quality of data was validated by routine use of internal quality controls, based on three different levels of proprietary materials.
NE-SSC, neutrophil cell complexity; NE-WX, neutrophil complexity and width of dispersion of the events measured; NE-WY, neutrophils fluorescence intensity and the width of dispersion; NE-WZ, neutrophil cell size and the width of dispersion; NRBCs, nucleated red blood cells; OMV, overall median; $\mathrm{RBC}$, red blood cell; $\mathrm{RCV}$, reference change values; RDW-CV, red blood cell distribution width; RT, room temperature; SFL, fluorescence intensity; SSC, side scatter; W, distribution width; WBC, white blood cell; XN-module, Sysmex XN-9000. 


\section{Within-run imprecision of cell population data}

Five blood samples collected in $\mathrm{K}_{3}$ EDTA tubes (Becton Dickinson, NJ) from a single healthy volunteer (the inclusion criteria were based on negative clinical history and normal serum concentrations of glucose, creatinine, aminotransferases, ferritin and $\mathrm{C}$ reactive protein) were preliminary mixed, and five identical aliquots were then produced. Within-run imprecision of CPD parameters was assessed by performing 10 consecutive measures of the five aliquots, as recommended by the Clinical and Laboratory Standards Institute (CLSI) document EP05-A3 (13). All aliquots were then analyzed in all the five separate $\mathrm{XN}$-modules with a specific protocol, as follows: in the first round the samples were analyzed from module 1 to 5 , in the second round from module 2 to 1 , up to the fifth round, when samples were analyzed from module 5 to 1 . The cycle was repeated twice.

The evaluation of imprecision was carried out by comparison with analytical performance specifications for imprecision $\left(\mathrm{CV}_{\text {APS }}\right)$ of $\mathrm{CPD}$ parameters, as described elsewhere by Buoro et al. (14).

\section{Verification of analyzers harmonization}

Venous blood samples were drawn from 30 apparently healthy blood donors (30-HBD) in $\mathrm{K}_{3}$ EDTA tubes in three different days (i.e., 10 samples per day over one month; November 2015), and then analyzed within 30 minutes from collection on each of the five $\mathrm{XN}$-modules. The inclusion criteria for these reference subjects were also based on a negative clinical history and normal serum concentrations of glucose, creatinine, aminotransferases, ferritin and $\mathrm{C}$ reactive protein.

The so-called Moving Average (MA) was assessed using all normal routine samples analyzed in the local laboratory over one month (i.e., November 2015). The inclusion criteria were as follows: absence of morphological flags, WBC count 4.0-10.0x $10 \% / \mathrm{L}$, hemoglobin $120-170 \mathrm{~g} / \mathrm{L}$, mean corpuscular volume (MCV) 84-98 fL, platelet count 150-450X $10^{9} / \mathrm{L}$, red blood cell (RBC) distribution width (RDW$\mathrm{CV})<14.0 \%$, nucleated RBCs (NRBCs) $<0.01 \times$ $10^{9} / \mathrm{L}$, NE count $2.00-5.60 \times 10^{9} / \mathrm{L}$, LY count 1.50 $3.50 \times 10^{9} / \mathrm{L}, \mathrm{MO}$ count $0.30-0.80 \times 10^{9} / \mathrm{L}$, eosinophil count $0.10-0.60 \times 10^{9} / \mathrm{L}$ and basophil count $<0.20 \times$ $10^{9} / \mathrm{L}$. According to these criteria, we finally selected 6,702 out of a total number of 35,500 routine samples $(18.9 \%)$ received in the laboratory during the 1month period. The daily results of normal level of the control material e-CHECK (XN-Check; Sysmex, Kobe, Japan) were also included in the evaluation. $\mathrm{XN}$-Check was repeated for 40 days, for a total of 40 measurements on each of the five $\mathrm{XN}$-modules. The median values of the CPD parameters obtained on each of the five $\mathrm{XN}$-modules of samples were then compared. The overall harmonization of CPD parameters was finally assessed by calculating the percentage bias (Bias\%) between the median value of results of each $\mathrm{XN}$-module and the median value of combined results obtained with all the five $\mathrm{XN}$-modules.

Due to the lack of internal and external quality controls for CPD parameters, we used the Overall Median (OMV) of each parameter as the reference value. The calculation of percentage difference between CPD parameters on each XN-Module was assessed using the formula [(median XN-Module OMV) / (Median XN-Module + OMV)/2)]*100, as suggested by Fraser et al. (15). The percent differences (Bias\%) were then compared to the analytical performance specification for Bias \% (B $\left.\mathrm{B}_{\mathrm{APS}} \%\right)$ reported elsewhere (14), whilst alignment bias based on intra-individual biological variation $\left(\mathrm{B}_{\mathrm{ALG}} \%\right.$ ) was calculated according to Petersen et al. (16-18), using the formula $0.33^{*} \mathrm{CV}_{1}$. The $\mathrm{CV}_{1}$ s were obtained from data earlier published by Buoro et al. (14). Difference significance of Bias\%, and between $\mathrm{B}_{\mathrm{APS}}$ and $\mathrm{B}_{\mathrm{ALG}}$ was assessed by estimating the overlap of $95 \%$ confidence interval $(\mathrm{Cl})$.

The distribution of data was tested with Shapiro and Wilk test (19). Results were compared with Kruskal-Wallis test and one-way analysis of variance (ANOVA).

Both MA, 30-HBD samples and XN-Check control blood assessment were repeated after two months (January 2016). The possibility of introducing an instrumental alignment factor (IAF) was tested, as follows: overall mean/mean value of $\mathrm{XN}$-module.

\section{Assessment of blood sample stability}

Ten ostensibly healthy, adult, Caucasian volunteers ( 5 women and 5 men; mean age $37 \pm 1$ years and $35 \pm 2$ years, respectively) were included in this part of the study. The inclusion criteria were again based on negative clinical history and normal serum concentrations of glucose, creatinine, aminotransferases, ferritin and $\mathrm{C}$ reactive protein. Venous blood samples (six samples for each subject) were drawn from an antecubital vein into $\mathrm{K}_{3}$ EDTA evacuated blood tubes. The samples were analyzed $15-30 \mathrm{~min}$ after the venipuncture (TO). Three tubes were stored at Room Temperature (RT), whilst the remaining three tubes drawn from each subject were aliquoted and kept refrigerated at $+4{ }^{\circ} \mathrm{C}$. With the aim of studying the effect of storage time, each sample was then repeatedly measured after 4 hours (T4), 6 hours (T6), 8 hours (T8), 24 hours (T24), 36 hours (T36), up to 48 hours (T48) from collection, respectively. All measurements were performed in duplicate.

The differences between the various parameters measured in the paired aliquots were then assessed 
with Steel-Dwass-Critchlow-Fligner test (20-22), with evaluation of Hodges-Lehmann location shift for multiple comparisons among different groups, after verification of values distribution with Shapiro-Wilk test. P-values $<0.05$ were considered statistically significant. The results were finally reported as $X\left(T_{X}-T_{0}\right)$ in absolute value.

Percentage variations from the baseline result (TO) in samples with statistically significant differences were then analyzed with Bland-Altman plots (B\%) and compared to $\mathrm{B}_{\text {APS }} \%$ (14). The Bias\% was also compared with the respective reference change values (RCV) (14).

\section{Statistical analysis}

The statistical analysis was performed using Analyse-it ${ }^{\mathrm{TM}}$ software, version 3.90.5 (Analyse-it software Ltd; Leeds, UK). The study was approved by the ethical committee of the Papa Giovanni XXIII Hospital and was carried out in accordance with the Declaration of Helsinki under the terms of all relevant local legislation.

\section{Results}

\section{Within-run imprecision}

The results of within-run imprecision (expressed as coefficient of variation; $\mathrm{CV}$ ) are shown in Table $I$. The imprecision of NE-parameters was $0.4-5.5 \%$, whilst that of LY-parameters was $0.7-8.7 \%$. Remarkable variability was noticed in the within-run imprecision of $\mathrm{MO}$-parameters across the five $\mathrm{XN}$-modules, being $0.8-14.1 \%$. Acceptable performance compared with the desirable analytical specification for imprecision derived from biological variation data published by Buoro et al. (14) was only met for NESFL and LYYY across the five XN-modules. Although all CPD parameters reflecting cellular dispersion did not even fulfill the minimum target of analytical imprecision, the other parameters achieved this target in most $\mathrm{XN}$-modules.

\section{Analyzers harmonization}

The mean values of the CPD parameters obtained in each group of samples are shown in Tables II, III, IV and Figures 1, 2 and 3. Albeit a good agreement was found among the different $\mathrm{XN}$-modules (maximum $4.0 \%$ Bias, except for NE, LY and MO-WZ), the median values were significantly different using both Kruskal-Wallis test and ANOVA, except for NE-WX, LY-WY, MO-WY.

The alignment was also assessed using intraindividual biological variation data (i.e., $\mathrm{CV}_{\mathrm{f}}$ ). The MO-WY parameter displayed a Bias\% lower than the $\mathrm{B}_{\mathrm{ALG}} \%$ obtained from biological variation $\left(0.33{ }^{*} \mathrm{CV}_{1}\right)$
(15) in all XN-Modules (Table II, III, IV). The comparison between the target $\mathrm{B}_{\mathrm{ALG}} \%$ and $\mathrm{B}_{\mathrm{APS}} \%$ (14) showed a significant difference (i.e., lack of overlap between $95 \% \mathrm{Cls}$ ) for only the NE-SSC, NE-FCS, NEWX, LY-WX and LY-WY parameters.

Notably, the median values of the CPD-parameters were not significantly different between the group of 30 healthy blood donors and the MA population. Nevertheless, the relative $\mathrm{B} \%$ recorded for each $\mathrm{XN}$ module using control blood (i.e., containing stabilized cells) was found to be similar to that observed using fresh blood (i.e., obtained from the 30 healthy blood donors and the MA population). The median values of the CPD parameters were found to be similar to those obtained in a subsequent study, which was repeated two months later using additional fresh blood from 30 healthy blood donors and the MA population. These data attest that CPD-parameters may be quite stable over time.

The IAF was then calculated for each CPD parameter, and for each of the five $\mathrm{XN}$-modules. When all CPD data were adjusted for the instrumentspecific IAF calculated from the MA population, the mean values were no longer statistically different among the five $\mathrm{XN}$-modules, as shown in Figures 1, 2 and 3 . In this case, the Bias\% of all parameters for each $\mathrm{XN}$-module was found to be lower than the $B_{A L G} \%$

Data adjustment for instrument-specific IAF calculated from the results obtained on blood collected from 30 healthy blood donors confirmed the data, thus obtaining the same effective harmonization (data not shown). Identical results were obtained when the identical study was repeated two months afterward, thus suggesting that IAF should only be calculated on data generated from an MA population.

\section{Assessment of blood sample stability}

The different CPD-parameters displayed heterogeneous stability, mostly depending on the storage temperature (Table VI). Overall, lower stability was observed in samples stored at $4{ }^{\circ} \mathrm{C}$. More specifically, 2 hours storage at $4{ }^{\circ} \mathrm{C}$ was already sufficient to impair the assessment of NE-FSC, NE-WX and MO$X$, whilst NE-SSC, NE-SFL, NE-WY, LY-Y, LY-WY, MO$Z$ and MO-WY were found to be stable for at least 8 hours, regardless of the storage temperature. Sample storage at $4{ }^{\circ} \mathrm{C}$ for 2 hours generated a $\mathrm{B} \%$ higher than the analytical performance specification for Bias $\left(B_{\text {APS }}\right)(14)$ for all parameters except NE-SFL, LY-Y, LYWY, and MO-WY. Nevertheless, the \%B of all parameters remained always lower than the RCV in all samples stored for up to 8 hours at both storage temperatures. 
Table I Within-run imprecision CPD parameters: the mean value (ch) and \%CV, obtained from 10 replicates of five aliquots of a healthy subject, are shown and compared $\mathrm{CV}_{\text {APS }}(14)$.

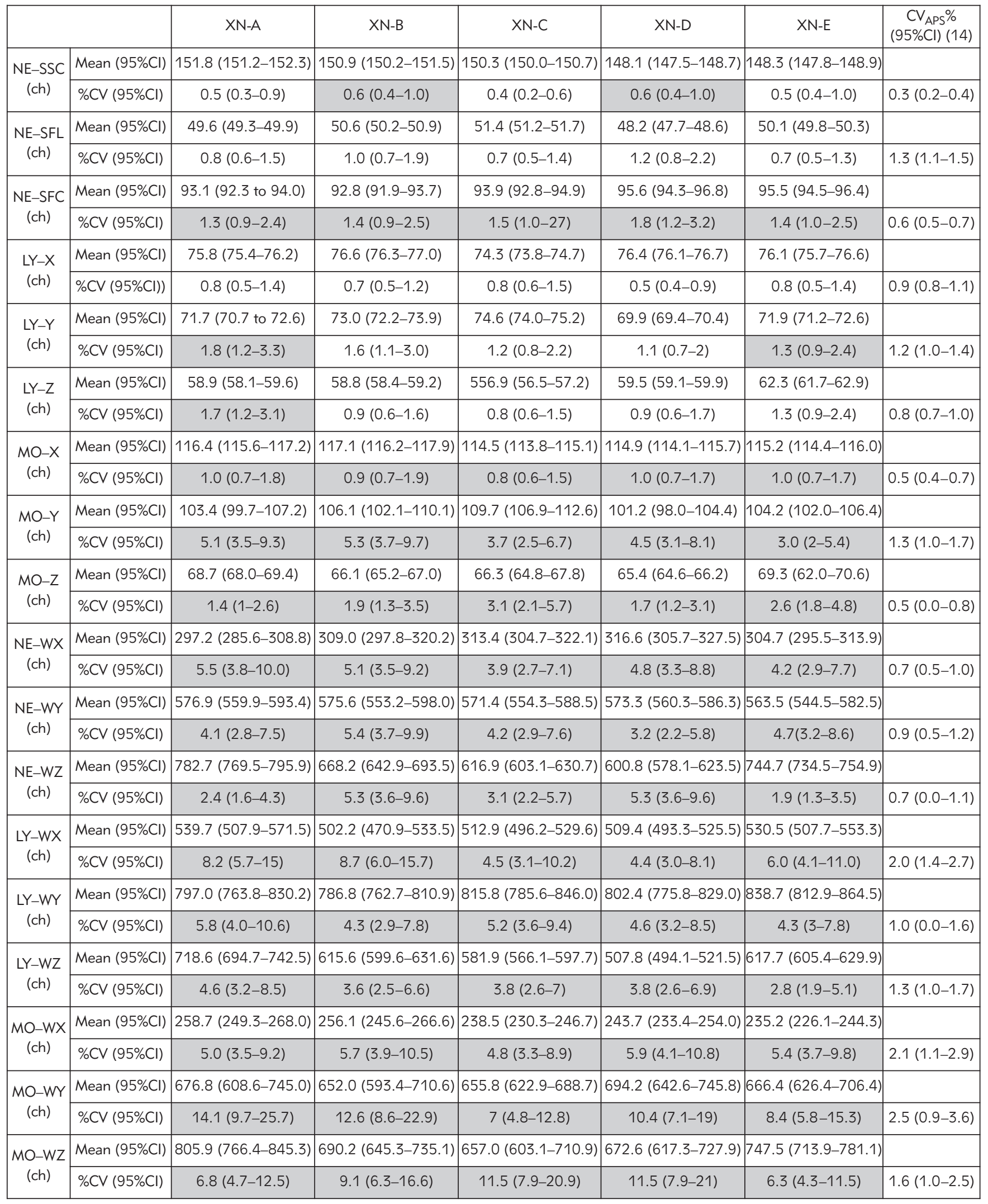

Legend: $\mathrm{CV}_{\text {APS }}$ : analytical performance specification for imprecision for desirable level; highlighted in grey: the imprecision does not meet the analytical goal for minimum performance. 
Table II Analyzers harmonization: lymphocytes cell population data. The median value and relative Bias (\%) (calculated with respect to overall median $[\mathrm{OMV}])$ of lymphocytes cell population data parameters to $\mathrm{B}_{\mathrm{APS}} \%(14)$ and target alignment Bias proposed by Fraser et al. $\left(0.33 \times \mathrm{CV}_{1}\right)\left(\mathrm{B}_{\mathrm{ALG}} \%\right)(15)$.

\begin{tabular}{|c|c|c|c|c|c|c|c|c|c|c|}
\hline & \multicolumn{3}{|c|}{$\begin{array}{l}30 \text { healthy blood donors } \\
\text { November } 2015\end{array}$} & \multicolumn{2}{|c|}{$\begin{array}{l}\text { Moving Average } \\
\text { November } 2015\end{array}$} & \multicolumn{2}{|c|}{$\begin{array}{l}\text { XN-Check (Normal level) } \\
\text { November } 2015\end{array}$} & \multirow{2}{*}{$\begin{array}{l}{ }_{\text {B APS } \%} \\
(95 \% \mathrm{Cl})\end{array}$} & \multirow{2}{*}{$\begin{array}{l}\mathrm{B}_{\mathrm{ALG}} \% \\
(95 \% \mathrm{Cl})\end{array}$} \\
\hline & & $\begin{array}{l}\text { Median } \\
(95 \% \mathrm{Cl})\end{array}$ & $\begin{array}{c}\text { Bias\% vs OVM } \\
(95 \% \mathrm{Cl})\end{array}$ & \begin{tabular}{c|}
$\mathrm{N}$ \\
samples
\end{tabular} & $\begin{array}{l}\text { Median } \\
(95 \% \mathrm{Cl})\end{array}$ & $\begin{array}{c}\text { Bias\% vs OVM } \\
(95 \% \mathrm{Cl})\end{array}$ & $\begin{array}{l}\text { Median } \\
(95 \% \mathrm{Cl})\end{array}$ & $\begin{array}{c}\text { Bias\% vs OVM } \\
(95 \% \mathrm{Cl})\end{array}$ & & \\
\hline \multirow{6}{*}{$\begin{array}{c}\text { NE-SSC } \\
\text { (ch) } \\
\S ¥\end{array}$} & XN-A & $\begin{array}{c}152.15 \\
(150.70 / 154.59)\end{array}$ & $\begin{array}{c}1.22 \\
(1.208 / 1.239)\end{array}$ & 1426 & $\begin{array}{c}152.10 \\
(151.9 / 152.4)\end{array}$ & $\begin{array}{c}1.14 \\
(1.139 / 1.142)\end{array}$ & $\begin{array}{c}170.05 \\
(169.80 / 170.30)\end{array}$ & $\begin{array}{c}1.18 \\
(1.178 / 1.182)\end{array}$ & \multirow{6}{*}{$\begin{array}{c}0.60 \\
(0.50 / 0.90)\end{array}$} & \multirow{6}{*}{$\begin{array}{l}0.20 \\
(0.16 / 0.23)\end{array}$} \\
\hline & $X N-B$ & $\begin{array}{c}149.20 \\
(147.50 / 151.80) \\
\end{array}$ & $\begin{array}{c}-0.75 \\
(-0.741 /-0.763)\end{array}$ & 1555 & $\begin{array}{c}149.40 \\
(149.10 / 149.70)\end{array}$ & $\begin{array}{c}-0.65 \\
(-0.649 /-0.651)\end{array}$ & $\begin{array}{c}166.95 \\
(166.80 / 167.10)\end{array}$ & $\begin{array}{c}-0.66 \\
(-0.659 /-0.661)\end{array}$ & & \\
\hline & $\mathrm{XN}-\mathrm{C}$ & $\begin{array}{c}151.60 \\
(149.30 / 153.50)\end{array}$ & $\begin{array}{c}0.85 \\
(0.837-0.861)\end{array}$ & 1687 & $\begin{array}{c}151.10 \\
(151.0 / 151.3)\end{array}$ & $\begin{array}{c}0.48 \\
(0.480 / 0.481)\end{array}$ & $\begin{array}{c}168.80 \\
(168.60 / 169.0)\end{array}$ & $\begin{array}{c}0.44 \\
(0.439 / 0.441) \\
\end{array}$ & & \\
\hline & $\mathrm{XN}-\mathrm{D}$ & \begin{tabular}{|c|}
149.70 \\
$(147.20 / 151.40)$
\end{tabular} & $\begin{array}{c}-0.41 \\
(-0.403 /-0.415)\end{array}$ & 1124 & $\begin{array}{c}150.00 \\
(149.6 / 150.3)\end{array}$ & $\begin{array}{c}-0.25 \\
(-0.249 /-0.251)\end{array}$ & $\begin{array}{c}167.40 \\
(167.30 / 167.60)\end{array}$ & $\begin{array}{c}-0.39 \\
(-0.389 /-0.391)\end{array}$ & & \\
\hline & $X N-E$ & $\begin{array}{c}148.95 \\
(146.90 / 150.60)\end{array}$ & $\begin{array}{c}-0.91 \\
(-0.897 /-0.920)\end{array}$ & 910 & $\begin{array}{c}149.30 \\
(148.8 / 149.6)\end{array}$ & $(-0.718 /-0.72$-0.721) & $\begin{array}{c}167.10 \\
(166.90 / 167.20)\end{array}$ & $\begin{array}{c}-0.57 \\
(-0.569 /-0.570)\end{array}$ & & \\
\hline & OVM & $\begin{array}{c}150.32 \\
(148.32 / 152.36)\end{array}$ & & & $\begin{array}{c}150.38 \\
(150.08 / 150.66)\end{array}$ & & $\begin{array}{c}168.06 \\
(167.88 / 168.24)\end{array}$ & & & \\
\hline \multirow{6}{*}{$\begin{array}{c}\text { NE-SFL } \\
\text { (ch) } \\
\S ¥\end{array}$} & XN-A & $\begin{array}{c}50.05 \\
(48.50 / 50.80)\end{array}$ & $\begin{array}{c}0.72 \\
(0.698 / 0.731)\end{array}$ & 1426 & $\begin{array}{c}49.30 \\
(49.10 / 49.50)\end{array}$ & $\begin{array}{c}1.07 \\
(1.066 / 1.074)\end{array}$ & $\begin{array}{c}99.70 \\
(99.20 / 100.20)\end{array}$ & $\begin{array}{c}1.23 \\
(1.224 / 1.236)\end{array}$ & \multirow{6}{*}{$-\left(\begin{array}{c}1.10 \\
(0.90 / 1.50)\end{array}\right.$} & \multirow{6}{*}{$\begin{array}{c}0.90 \\
(0.76 / 1.02)\end{array}$} \\
\hline & $X N-B$ & $\begin{array}{c}48.85 \\
(47.50 / 50.00)\end{array}$ & $\begin{array}{c}-1.69 \\
(-1.643 /-1.730)\end{array}$ & 1555 & $\begin{array}{c}48.40 \\
(48.30 / 48.60)\end{array}$ & $\begin{array}{c}-0.78 \\
(-0.778 /-0.783)\end{array}$ & $\begin{array}{c}95.75 \\
(95.40 / 96.90)\end{array}$ & $\begin{array}{c}-2.78 \\
(-2.770 /-2.813)\end{array}$ & & \\
\hline & $\mathrm{XN}-\mathrm{C}$ & $\begin{array}{c}50.90 \\
(49.10 / 51.50)\end{array}$ & $\begin{array}{c}2.44 \\
(2.354 / 2.469)\end{array}$ & 1687 & $\begin{array}{c}50.60 \\
(50.50 / 50.70)\end{array}$ & $\begin{array}{c}3.73 \\
(3.723 / 3.737)\end{array}$ & $\begin{array}{c}102.50 \\
(102.30 / 103.60)\end{array}$ & $\begin{array}{c}4.07 \\
(4.062 / 4.114)\end{array}$ & & \\
\hline & $X N-D$ & $\begin{array}{c}48.30 \\
(46.90 / 48.70)\end{array}$ & $\begin{array}{c}-2.80 \\
(-2.719 /-2.823)\end{array}$ & 1124 & $\begin{array}{c}46.90 \\
(46.70 / 47.10)\end{array}$ & $\begin{array}{c}-3.85 \\
(-3.834 /-3.866)\end{array}$ & $\begin{array}{c}97.00 \\
(96.70 / 97.30)\end{array}$ & $\begin{array}{c}-1.51 \\
(-1.505 /-1.515)\end{array}$ & & \\
\hline & XN-E & $\begin{array}{c}50.35 \\
(49.00 / 51.40)\end{array}$ & $\begin{array}{c}1.33 \\
(1.294 / 1.358)\end{array}$ & 910 & $\begin{array}{c}48.70 \\
(48.50 / 48.80) \\
\end{array}$ & $\begin{array}{c}-0.16 \\
(-0.159 /-0.160)\end{array}$ & $\begin{array}{c}97.50 \\
(97.20798 .00) \\
\end{array}$ & $\begin{array}{c}-1.01 \\
(-1.007 /-1.015)\end{array}$ & & \\
\hline & OVM & $\begin{array}{c}49.69 \\
(48.20 / 50.48)\end{array}$ & & & $\begin{array}{c}48.78 \\
(48.62 / 48.94)\end{array}$ & & $\begin{array}{c}98.49 \\
(98.16 / 99.20)\end{array}$ & & & \\
\hline \multirow{6}{*}{$\begin{array}{c}\text { NE-FCS } \\
\text { (ch) } \\
\S ¥\end{array}$} & XN-A & $\begin{array}{c}90.90 \\
(89.10 / 92.20)\end{array}$ & $\begin{array}{c}0.69 \\
(0.676 / 0.700)\end{array}$ & 1426 & $\begin{array}{c}89.60 \\
(89.30 / 89.80)\end{array}$ & $\begin{array}{c}0.43 \\
(0.429 / 0.431)\end{array}$ & $\begin{array}{c}124.95 \\
(124.20 / 125.30)\end{array}$ & $\begin{array}{c}0.85 \\
(0.845 / 0.852)\end{array}$ & \multirow{6}{*}{$-(0.60 / 1.10)$} & \multirow{6}{*}{$\begin{array}{c}0.40 \\
(0.33 / 0.50\end{array}$} \\
\hline & $X N-B$ & $\begin{array}{c}89.05 \\
(87.60 / 89.70)\end{array}$ & $(-1.338 /-1.370)$ & 1555 & $\begin{array}{c}87.70 \\
(87.60 / 88.00)\end{array}$ & $\begin{array}{c}-1.70 \\
(-1.698 /-1.706)\end{array}$ & $\begin{array}{c}120.45 \\
(119.60 / 121.10)\end{array}$ & $\begin{array}{c}-2.78 \\
(-2.760 /-2.795)\end{array}$ & & \\
\hline & $\mathrm{XN}-\mathrm{C}$ & $\begin{array}{c}88.75 \\
(87.30 / 89.70)\end{array}$ & $\begin{array}{c}-1.69 \\
(-1.662 /-1.708)\end{array}$ & 1687 & $\begin{array}{c}88.30 \\
(88.10 / 88.50)\end{array}$ & $\begin{array}{c}-1.03 \\
(-1.028 /-1.032)\end{array}$ & $\begin{array}{c}123.50 \\
(123.10 / 123.80)\end{array}$ & $\begin{array}{c}-0.32 \\
(-0.319 /-0.321)\end{array}$ & & \\
\hline & XN-D & $\begin{array}{c}91.00 \\
(89.70 / 92.40)\end{array}$ & $\begin{array}{c}0.80 \\
(0.789 / 0.812)\end{array}$ & 1124 & $\begin{array}{c}90.40 \\
(90.20 / 90.60)\end{array}$ & $\begin{array}{c}1.32 \\
(1.317 / 1.323)\end{array}$ & $\begin{array}{c}125.30 \\
(124.90 / 125.60)\end{array}$ & $\begin{array}{c}1.13 \\
(1.126 / 1.133)\end{array}$ & & \\
\hline & $X N-E$ & $\begin{array}{c}91.70 \\
(89.80 / 92.70)\end{array}$ & $\begin{array}{c}1.57 \\
(1.537 / 1.587)\end{array}$ & 910 & $\begin{array}{c}90.10 \\
(89.90 / 90.40)\end{array}$ & $\begin{array}{c}0.99 \\
(0.988 / 0.993)\end{array}$ & $\begin{array}{c}125.30 \\
(125.00 / 125.60)\end{array}$ & $\begin{array}{c}1.13 \\
(1.127 / 1.133)\end{array}$ & & \\
\hline & OVM & $\begin{array}{c}90.28 \\
(88.70 / 91.34)\end{array}$ & & & $\begin{array}{c}89.22 \\
(89.02 / 89.46)\end{array}$ & & $\begin{array}{c}123.90 \\
(123.36 / 124.28)\end{array}$ & & & \\
\hline \multirow{6}{*}{$\begin{array}{l}\text { NE-WX } \\
\text { (ch) }\end{array}$} & XN-A & $\begin{array}{c}314.00 \\
(309.00 / 320.00)\end{array}$ & $\begin{array}{c}1.00 \\
(0.984 / 1.019)\end{array}$ & 1426 & $\begin{array}{c}312.00 \\
(311.00 / 313.00)\end{array}$ & $\begin{array}{c}1.01 \\
(1.007 / 1.013)\end{array}$ & $\begin{array}{c}332.00 \\
(332.00 / 339.00)\end{array}$ & $\begin{array}{c}1.02 \\
(1.020 / 1.042)\end{array}$ & \multirow{6}{*}{$\begin{array}{c}0.80 \\
(0.60 / 1.20)\end{array}$} & \multirow{6}{*}{$\begin{array}{c}0.40 \\
(0.00 / 0.66)\end{array}$} \\
\hline & $X N-B$ & \begin{tabular}{|c|}
316.00 \\
$(312.00 / 320.00)$
\end{tabular} & $\begin{array}{c}1.00 \\
(0.987 / 1.013)\end{array}$ & 1555 & $\begin{array}{c}319.00 \\
(318.00 / 319.00)\end{array}$ & $\begin{array}{c}0.99 \\
(0.987 / 0.990)\end{array}$ & $\begin{array}{c}341.50 \\
(336.00 / 342.00)\end{array}$ & $\begin{array}{c}0.99 \\
(0.974 / 0.991)\end{array}$ & & \\
\hline & $\mathrm{XN}-\mathrm{C}$ & \begin{tabular}{|c|}
320.50 \\
$(311.00 / 323.00)$
\end{tabular} & $\begin{array}{c}0.98 \\
(0.951 / 0.988)\end{array}$ & 1687 & $\begin{array}{c}317.00 \\
(316.00 / 318.00)\end{array}$ & $\begin{array}{c}1.00 \\
(0.997 / 1.003)\end{array}$ & $\begin{array}{c}343.00 \\
(337.00 / 343.00)\end{array}$ & $\begin{array}{c}0.99 \\
(0.973 / 0.990)\end{array}$ & & \\
\hline & $X N-D$ & $\begin{array}{c}314.00 \\
(309.00 / 324.00)\end{array}$ & $\begin{array}{c}1.00 \\
(0.984 / 1.032)\end{array}$ & 1124 & $\begin{array}{c}317.00 \\
(316.00 / 318.00)\end{array}$ & $\begin{array}{c}1.00 \\
(0.997 / 1.003)\end{array}$ & $\begin{array}{c}340.50 \\
(335.00 / 341.00)\end{array}$ & $\begin{array}{c}1.00 \\
(0.984 / 1.001)\end{array}$ & & \\
\hline & $X N-E$ & $\begin{array}{c}309.00 \\
(303.00 / 323.00)\end{array}$ & $\begin{array}{c}1.02 \\
(1.000 / 1.066)\end{array}$ & 910 & $\begin{array}{c}314.00 \\
(312.00 / 315.00)\end{array}$ & $\begin{array}{c}1.01 \\
(1.004 / 1.013)\end{array}$ & $\begin{array}{c}340.00 \\
(335.00 / 340.00)\end{array}$ & $\begin{array}{c}1.00 \\
(0.985 / 1.000)\end{array}$ & & \\
\hline & OVM & $\begin{array}{c}314.70 \\
(308.80 / 322.00)\end{array}$ & & & $\begin{array}{c}315.80 \\
(314.60 / 316.60)\end{array}$ & & $\begin{array}{c}339.30 \\
(335.00 / 341.00\end{array}$ & & & \\
\hline \multirow{6}{*}{$\begin{array}{c}\text { NE-WY } \\
\text { (ch) } \\
\S ¥\end{array}$} & XN-A & \begin{tabular}{|c|}
592.00 \\
$(580.00 / 596.00)$
\end{tabular} & $\begin{array}{c}1.01 \\
(0.990 / 1.017)\end{array}$ & 1426 & $\begin{array}{c}585.00 \\
(584.00 / 587.00)\end{array}$ & $\begin{array}{c}1.00 \\
(0.998 / 1.003)\end{array}$ & $\begin{array}{c}668.50 \\
(652.00 / 679.00)\end{array}$ & $\begin{array}{c}1.00 \\
(0.975 / 1.016)\end{array}$ & \multirow{6}{*}{$\begin{array}{c}0.50 \\
(0.30 / 0.80)\end{array}$} & \multirow{6}{*}{$\begin{array}{c}0.60 \\
(0.33 / 0.81)\end{array}$} \\
\hline & $X N-B$ & \begin{tabular}{|c|}
591.00 \\
$(582.00 / 600.00)$
\end{tabular} & $\begin{array}{c}1.01 \\
(0.995 / 1.025)\end{array}$ & 1555 & $\begin{array}{c}591.00 \\
(589.00 / 592.00)\end{array}$ & $\begin{array}{c}0.99 \\
(0.987 / 0.992)\end{array}$ & $\begin{array}{c}670.50 \\
(655.00 / 686.00)\end{array}$ & $\begin{array}{c}1.00 \\
(0.977 / 1.023)\end{array}$ & & \\
\hline & $\mathrm{XN}-\mathrm{C}$ & \begin{tabular}{|c|}
591.00 \\
$(577.00 / 605.00)$
\end{tabular} & $\begin{array}{c}1.01 \\
(0.986 / 1.034)\end{array}$ & 1687 & $\begin{array}{c}588.00 \\
(586.00 / 590.00)\end{array}$ & $\begin{array}{c}1.00 \\
(0.997 / 1.003)\end{array}$ & $\begin{array}{c}663.00 \\
(648.00 / 678.00)\end{array}$ & $\begin{array}{c}1.01 \\
(0.987 / 1.033)\end{array}$ & & \\
\hline & XN-D & $\begin{array}{c}602.50 \\
(599.00 / 613.00)\end{array}$ & $\begin{array}{c}0.99 \\
(0.984 / 1.007)\end{array}$ & 1124 & $\begin{array}{c}594.00 \\
(592.00 / 596.00)\end{array}$ & $\begin{array}{c}0.99 \\
(0.987 / 0.993)\end{array}$ & $\begin{array}{c}666.50 \\
(653.00 / 672.00)\end{array}$ & $\begin{array}{c}1.00 \\
(0.980 / 1.008)\end{array}$ & & \\
\hline & $X N-E$ & $\begin{array}{c}604.00 \\
(588.00 / 610.00)\end{array}$ & $\begin{array}{c}0.99 \\
(0.964 / 1.000)\end{array}$ & 910 & $\begin{array}{c}579.00 \\
(577.00 / 581.00)\end{array}$ & $\begin{array}{c}1.01 \\
(1.007 / 1.013)\end{array}$ & $\begin{array}{c}669.00 \\
(649.00 / 677.00)\end{array}$ & $\begin{array}{c}1.00 \\
(0.970 / 1.012)\end{array}$ & & \\
\hline & OVM & $\begin{array}{c}596.10 \\
(585.20 / 604.80)\end{array}$ & & & $\begin{array}{c}587.40 \\
(585.60 / 589.20)\end{array}$ & & $\begin{array}{c}667.60 \\
(651.40 / 678.40)\end{array}$ & & & \\
\hline \multirow{6}{*}{$\begin{array}{c}\text { NE-WZ } \\
\text { (ch) } \\
\$ ¥\end{array}$} & XN-A & $\begin{array}{c}793.50 \\
(785.00 / 802.00)\end{array}$ & $\begin{array}{c}8.80 \\
(8.706 / 8.894)\end{array}$ & 1426 & $\begin{array}{c}786.00 \\
(785.00 / 789.00)\end{array}$ & $\begin{array}{c}9.62 \\
(9.608 / 9.657)\end{array}$ & $\begin{array}{c}835.00 \\
(828.00 / 849.00)\end{array}$ & $\begin{array}{c}-1.66 \\
(-1.649 /-1.691)\end{array}$ & \multirow{6}{*}{$(0.30 / 1.00)$} & \multirow{6}{*}{$\begin{array}{c}0.50 \\
(0.00 / 0.73)\end{array}$} \\
\hline & $\mathrm{XN}-\mathrm{B}$ & $\begin{array}{c}740.50 \\
(727.00 / 753.00)\end{array}$ & $\begin{array}{c}1.53 \\
(1.502 / 1.556)\end{array}$ & 1555 & $\begin{array}{c}727.00 \\
(725.00 / 730.00)\end{array}$ & $\begin{array}{c}1.39 \\
(1.386 / 1.396)\end{array}$ & $\begin{array}{c}849.00 \\
(822.00 / 859.00)\end{array}$ & $\begin{array}{c}7.33 \\
(7.093 / 7.412)\end{array}$ & & \\
\hline & $\mathrm{XN}-\mathrm{C}$ & $\begin{array}{c}676.00 \\
(666.00 / 697.00)\end{array}$ & $\begin{array}{c}-7.30 \\
(-7.192 /-7.527)\end{array}$ & 1687 & $\begin{array}{c}671.00 \\
(669.00 / 673.00)\end{array}$ & $\begin{array}{c}-6.42 \\
(-6.401 /-6.439)\end{array}$ & $\begin{array}{c}789.00 \\
(769.00 / 805.00)\end{array}$ & $\begin{array}{c}6.68 \\
(6.510 / 6.815)\end{array}$ & & \\
\hline & XN-D & \begin{tabular}{|c|}
636.00 \\
$(613.00 / 650.00)$
\end{tabular} & $(-12.327 /-12.79 .072)$ & 1124 & $\begin{array}{c}627.00 \\
(625.00 / 629.00)\end{array}$ & $\begin{array}{c}-12.55 \\
(-12.51 /-12.590)\end{array}$ & $\begin{array}{c}738.00 \\
(723.00 / 757.00)\end{array}$ & $\begin{array}{c}-18.73 \\
(-18.348 /-19.211)\end{array}$ & & \\
\hline & $X N-E$ & \begin{tabular}{|c|}
800.50 \\
$(782.00 / 821.00)$
\end{tabular} & $\begin{array}{c}9.76 \\
(9.534 / 10.010)\end{array}$ & 910 & $\begin{array}{c}774.00 \\
(770.00 / 776.00)\end{array}$ & $\begin{array}{c}7.95 \\
(7.909 / 7.971)\end{array}$ & $\begin{array}{c}890.50 \\
(881.00 / 916.00)\end{array}$ & $\begin{array}{c}8.21 \\
(8.119 / 8.442)\end{array}$ & & \\
\hline & OVM & $\begin{array}{c}729.30 \\
(714.60 / 744.60)\end{array}$ & & & $\begin{array}{c}717.00 \\
(714.80 / 719.40)\end{array}$ & & $\begin{array}{c}820.30 \\
(804.6 / 837.2)\end{array}$ & & & \\
\hline
\end{tabular}

§: comparison of median value of CPD parameter evaluated for all $5 \mathrm{XN}$ showed a significative difference in all pair comparisons by Kruskal-Wallis test with $\mathrm{p}<0.0001$ in all groups of samples; $¥$ : comparison of mean value of CPD parameter evaluated for all $5 \mathrm{XN}$ showed a significative difference on ANOVA with $\mathrm{p}<0.0001$ in all groups of samples. Highlighted in grey: Bias\% is lower than the $\mathrm{B}_{\mathrm{ALG}} \%$. $\mathrm{B}_{\mathrm{ALG}}$ : alignment bias based on intra-individual biological variation; $\mathrm{B}_{\mathrm{APS}}$ : analytical performance specification for Bias\%; NE-FSC: neutrophil cell size; NE-SFL: neutrophil fluorescence intensity; NE-SSC: neutrophil cell complexity; NE-WX: neutrophil complexity and width of dispersion of the events measured; NE-WY: neutrophils fluorescence intensity and the width of dispersion; NE-WZ: neutrophil cell size and the width of dispersion; OMV: overall median. 
Table III Analyzers harmonization: lymphocytes cell population data. The median value and relative Bias (\%) (in respect to overall median [OMV]) of lymphocytes cell population data parameters compared in respect to $B_{A P S} \%(14)$ and target alignment Bias proposed by Fraser et al. (0.33 $\left.\times C V I\right)\left(B_{A L G} \%\right)(15)$.

\begin{tabular}{|c|c|c|c|c|c|c|c|c|c|c|}
\hline & \multicolumn{3}{|c|}{$\begin{array}{l}30 \text { healthy blood donors } \\
\text { November } 2015\end{array}$} & \multicolumn{2}{|c|}{$\begin{array}{l}\text { Moving Average } \\
\text { November } 2015\end{array}$} & \multicolumn{2}{|c|}{$\begin{array}{l}\text { XN-Check (Normal level) } \\
\text { November } 2015\end{array}$} & \multirow{2}{*}{$\begin{array}{l}\mathrm{B}_{\mathrm{APS}} \% \\
(95 \% \mathrm{Cl})\end{array}$} & \multirow{2}{*}{$\begin{array}{l}\mathrm{B}_{\mathrm{ALG}} \% \\
(95 \% \mathrm{Cl})\end{array}$} \\
\hline & & $\begin{array}{l}\text { Median } \\
(95 \% \mathrm{Cl})\end{array}$ & $\begin{array}{c}\text { Bias\% vs OVM } \\
(95 \% \mathrm{Cl})\end{array}$ & $\begin{array}{c}\mathrm{N} \\
\text { samples }\end{array}$ & $\begin{array}{l}\text { Median } \\
(95 \% \mathrm{Cl})\end{array}$ & $\begin{array}{c}\text { Bias\% vs OVM } \\
(95 \% \mathrm{Cl})\end{array}$ & $\begin{array}{l}\text { Median } \\
(95 \% \mathrm{Cl})\end{array}$ & $\begin{array}{c}\text { Bias\% vs OVM } \\
(95 \% \mathrm{Cl})\end{array}$ & & \\
\hline \multirow{6}{*}{$\begin{array}{c}\text { LY-X } \\
\text { (ch) } \\
\S ¥\end{array}$} & $X N-A$ & $\begin{array}{c}78.45 \\
(77.50 / 78.90)\end{array}$ & $\begin{array}{c}1.76 \\
(1.739 / 1.770)\end{array}$ & 1426 & $\begin{array}{c}77.60 \\
(77.50 / 77.70)\end{array}$ & $\begin{array}{c}2.05 \\
(2.047 / 2.053)\end{array}$ & $\begin{array}{c}86.65 \\
(86.10 / 87.00)\end{array}$ & $\begin{array}{c}0.36 \\
(0.358 / 0.361)\end{array}$ & \multirow{6}{*}{$\begin{array}{c}0.70 \\
(0.50 / 0.90)\end{array}$} & \multirow{6}{*}{$\begin{array}{c}0.60 \\
(0.53 / 0.73)\end{array}$} \\
\hline & $X N-B$ & $\begin{array}{c}76.50 \\
(76.00 / 76.80)\end{array}$ & $\begin{array}{c}-0.77 \\
(-0.765 /-0.773)\end{array}$ & 1555 & $\begin{array}{c}75.50 \\
(75.30 / 75.60) \\
\end{array}$ & $\begin{array}{c}-0.71 \\
(-0.708 /-0.711)\end{array}$ & $\begin{array}{c}85.80 \\
(85.50 / 86.30) \\
\end{array}$ & $\begin{array}{c}-0.14 \\
(-0.140 /-0.141)\end{array}$ & & \\
\hline & $\mathrm{XN}-\mathrm{C}$ & $\begin{array}{c}75.75 \\
(75.20 / 76.60)\end{array}$ & $\begin{array}{c}-1.74 \\
(-1.727 /-1.760)\end{array}$ & 1687 & $\begin{array}{c}75.20 \\
(75.10 / 75.20)\end{array}$ & $\begin{array}{c}-1.10 \\
(-1.099 /-1.100)\end{array}$ & $\begin{array}{c}85.50 \\
(85.40 / 85.80)\end{array}$ & $\begin{array}{c}-0.32 \\
(-0.320 /-0.321)\end{array}$ & & \\
\hline & XN-D & $\begin{array}{c}77.35 \\
(76.50 / 77.90)\end{array}$ & $\begin{array}{c}0.34 \\
(0.336 / 0.342)\end{array}$ & 1124 & $\begin{array}{c}75.80 \\
(75.70 / 76.00)\end{array}$ & $\begin{array}{c}-0.32 \\
(-0.320 /-0.321)\end{array}$ & $\begin{array}{c}86.25 \\
(85.80 / 86.50)\end{array}$ & $\begin{array}{c}0.12 \\
(0.119 / 0.120)\end{array}$ & & \\
\hline & $X N-E$ & $\begin{array}{c}77.40 \\
(76.80 / 77.70)\end{array}$ & $\begin{array}{c}0.40 \\
(0.397 / 0.402)\end{array}$ & 910 & $\begin{array}{c}76.10 \\
(75.90 / 76.20)\end{array}$ & $\begin{array}{c}0.08 \\
(0.080 / 0.080)\end{array}$ & $\begin{array}{c}86.00 \\
(85.50 / 86.50)\end{array}$ & $\begin{array}{c}-0.02 \\
(-0.020 /-0.020)\end{array}$ & & \\
\hline & OVM & $\begin{array}{c}77.09 \\
(76.40 / 77.58)\end{array}$ & & & $\begin{array}{c}76.04 \\
(75.90 / 76.14)\end{array}$ & & $\begin{array}{c}86.04 \\
(85.66 / 86.42)\end{array}$ & & & \\
\hline \multirow{6}{*}{$\begin{array}{l}\text { LY-Y } \\
\text { (ch) } \\
\S ¥\end{array}$} & $X N-A$ & $\begin{array}{c}71.95 \\
(70.50 / 73.20)\end{array}$ & $\begin{array}{c}0.63 \\
(0.617 / 0.641)\end{array}$ & 1426 & $\begin{array}{c}72.00 \\
(71.90 / 72.20)\end{array}$ & $\begin{array}{c}1.21 \\
(1.208 / 1.213)\end{array}$ & $\begin{array}{c}67.75 \\
(67.40 / 68.20)\end{array}$ & $\begin{array}{c}-0.53 \\
(-0.527 /-0.534)\end{array}$ & \multirow{6}{*}{$\begin{array}{c}0.80 \\
(0.60 / 1.10)\end{array}$} & \multirow{6}{*}{$\begin{array}{c}0.76 \\
(0.63 / 0.92)\end{array}$} \\
\hline & $X N-B$ & $\begin{array}{c}70.45 \\
(69.50 / 71.40)\end{array}$ & $\begin{array}{c}-1.40 \\
(-1.381 /-1.419)\end{array}$ & 1555 & $\begin{array}{c}70.50 \\
(70.40 / 70.80)\end{array}$ & $\begin{array}{c}-0.90 \\
(-0.899 /-0.904)\end{array}$ & $\begin{array}{c}66.40 \\
(66.00 / 66.809\end{array}$ & $\begin{array}{c}-2.51 \\
(-2.495 /-2.525)\end{array}$ & & \\
\hline & $\mathrm{XN}-\mathrm{C}$ & $\begin{array}{c}72.70 \\
(71.10 / 74.10\end{array}$ & $\begin{array}{c}1.68 \\
(1.643 / 1.712)\end{array}$ & 1687 & $\begin{array}{c}73.80 \\
(73.60 / 73.90)\end{array}$ & $\begin{array}{c}3.74 \\
(3.730 / 3.745)\end{array}$ & $\begin{array}{c}70.60 \\
(70.20 / 70.80)\end{array}$ & $\begin{array}{c}3.66 \\
(3.639 / 3.670)\end{array}$ & & \\
\hline & $X N-D$ & $\begin{array}{c}70.10 \\
(69.00 / 70.50)\end{array}$ & $\begin{array}{c}-1.96 \\
(-1.929 /-1.971)\end{array}$ & 1124 & $\begin{array}{c}68.90 \\
(68.70 / 69.10)\end{array}$ & $\begin{array}{c}-3.15 \\
(-3.141 /-3.159)\end{array}$ & $\begin{array}{c}67.60 \\
(67.20 / 68.00)\end{array}$ & $\begin{array}{c}-0.75 \\
(-0.746 /-0.754)\end{array}$ & & \\
\hline & $X N-E$ & $\begin{array}{c}72.30 \\
(71.50 / 73.10)\end{array}$ & $\begin{array}{c}1.12 \\
(1.108 / 1.132)\end{array}$ & 910 & $\begin{array}{c}70.50 \\
(70.40 / 70.80)\end{array}$ & $\begin{array}{c}-0.90 \\
(-0.899 /-0.904)\end{array}$ & $\begin{array}{c}68.20 \\
(67.90 / 68.40)\end{array}$ & $\begin{array}{c}0.13 \\
(0.129 / 0.130)\end{array}$ & & \\
\hline & OVM & $\begin{array}{c}71.50 \\
(70.32 / 72.46)\end{array}$ & & & $\begin{array}{c}71.14 \\
(71.00 / 71.36)\end{array}$ & & $\begin{array}{c}68.11 \\
(67.74 / 68.44)\end{array}$ & & & \\
\hline \multirow{6}{*}{$\begin{array}{c}\text { LY-Z } \\
\text { (ch) } \\
\S \neq\end{array}$} & $X N-A$ & $\begin{array}{c}59.60 \\
(58.70 / 60.00)\end{array}$ & $\begin{array}{c}1.60 \\
(1.576 / 1.611)\end{array}$ & 1426 & $\begin{array}{c}59.80 \\
(59.70 / 59.80)\end{array}$ & $\begin{array}{c}1.98 \\
(1.977 / 1.980)\end{array}$ & $\begin{array}{c}91.35 \\
(90.80 / 91.80)\end{array}$ & $\begin{array}{c}-1.53 \\
(-1.521 /-1.538)\end{array}$ & \multirow{6}{*}{$\begin{array}{c}0.50 \\
(0.40 / 0.60)\end{array}$} & \multirow{6}{*}{$\begin{array}{c}0.53 \\
(0.43 / 0.63)\end{array}$} \\
\hline & $X N-B$ & $\begin{array}{c}58.15 \\
(57.80 / 58.60)\end{array}$ & $\begin{array}{c}-0.87 \\
(-0.865 /-0.877)\end{array}$ & 1555 & $\begin{array}{c}58.20 \\
(58.10 / 58.20)\end{array}$ & $\begin{array}{c}-0.75 \\
(-0.749 /-0.750)\end{array}$ & $\begin{array}{c}90.70 \\
(90.00 / 91.20)\end{array}$ & $\begin{array}{c}-2.23 \\
(-2.213 /-2.242)\end{array}$ & & \\
\hline & $\mathrm{XN}-\mathrm{C}$ & $\begin{array}{c}55.75 \\
(55.40 / 55.90)\end{array}$ & $\begin{array}{c}-4.96 \\
(-4.929 /-4.973)\end{array}$ & 1687 & $\begin{array}{c}56.50 \\
(56.40 / 56.60)\end{array}$ & $\begin{array}{c}-3.65 \\
(-3.644 /-3.656)\end{array}$ & $\begin{array}{c}90.90 \\
(90.70 / 91.40)\end{array}$ & $\begin{array}{c}-2.02 \\
(-2.016 /-2.031)\end{array}$ & & \\
\hline & $X N-D$ & $\begin{array}{c}58.20 \\
(57.60 / 58.50)\end{array}$ & $\begin{array}{c}-0.78 \\
(-0.772 /-0.784)\end{array}$ & 1124 & $\begin{array}{c}58.10 \\
(58.00 / 58.20)\end{array}$ & $\begin{array}{c}-0.92 \\
(-0.918 /-0.922)\end{array}$ & $\begin{array}{c}95.80 \\
(95.40 / 96.30)\end{array}$ & $\begin{array}{c}3.27 \\
(3.256 / 3.287)\end{array}$ & & \\
\hline & $X N-E$ & $\begin{array}{c}61.60 \\
(61.40 / 62.40)\end{array}$ & $\begin{array}{c}5.01 \\
(4.994 / 5.075)\end{array}$ & 910 & $\begin{array}{c}60.60 \\
(60.50 / 60.80)\end{array}$ & $\begin{array}{c}3.34 \\
(3.334 / 3.351)\end{array}$ & $\begin{array}{c}95.10 \\
(94.70 / 95.40)\end{array}$ & $\begin{array}{c}2.51 \\
(2.499 / 2.518)\end{array}$ & & \\
\hline & OVM & $\begin{array}{c}58.66 \\
(58.18 / 59.08)\end{array}$ & & & $\begin{array}{c}58.64 \\
(58.54 / 58.72)\end{array}$ & & $\begin{array}{c}92.77 \\
(92.32 / 93.22)\end{array}$ & & & \\
\hline \multirow{6}{*}{$\begin{array}{c}\text { LY-WX } \\
\text { (ch) } \\
\S ¥\end{array}$} & $X N-A$ & $\begin{array}{c}518.00 \\
(505.00 / 523.00)\end{array}$ & $\begin{array}{c}3.02 \\
(2.944 / 3.049)\end{array}$ & 1426 & $\begin{array}{c}519.0 \\
(515.00 / 521.00)\end{array}$ & $\begin{array}{c}1.65 \\
(1.637 / 1.656)\end{array}$ & $\begin{array}{c}703.00 \\
(699.00 / 708.00)\end{array}$ & $\begin{array}{c}-1.99 \\
(-1.979 /-2.004)\end{array}$ & \multirow{6}{*}{$\begin{array}{c}1.80 \\
(1.30 / 2.70)\end{array}$} & \multirow{6}{*}{$\begin{array}{c}1.32 \\
(0.91 / 1.75)\end{array}$} \\
\hline & $X N-B$ & $\begin{array}{c}503.50 \\
(478.00 / 527.00) \\
\end{array}$ & $\begin{array}{c}0.14 \\
(0.133 / 0.147) \\
\end{array}$ & 1555 & $\begin{array}{c}509.0 \\
(515.00 / 521.00)\end{array}$ & $\begin{array}{c}-0.31 \\
(-0.308 /-0.311)\end{array}$ & $\begin{array}{c}715.50 \\
(708.00 / 725.00) \\
\end{array}$ & $\begin{array}{c}-0.76 \\
(-0.752 /-0.770)\end{array}$ & & \\
\hline & $X N-C$ & $\begin{array}{c}520.00 \\
(490.00 / 546.00)\end{array}$ & $\begin{array}{c}3.42 \\
(3.223 / 3.591)\end{array}$ & 1687 & $\begin{array}{c}518.0 \\
(506.00 / 511.00)\end{array}$ & $\begin{array}{c}1.45 \\
(1.442 / 1.458)\end{array}$ & $\begin{array}{c}729.00 \\
(724.00 / 734.00)\end{array}$ & $\begin{array}{c}2.05 \\
(2.036 / 2.064)\end{array}$ & & \\
\hline & $X N-D$ & $\begin{array}{c}478.50 \\
(470.00 / 492.00)\end{array}$ & $\begin{array}{c}-4.83 \\
(-4.744 /-4.966)\end{array}$ & 1124 & $\begin{array}{c}505.00 \\
(515.00 / 521.00)\end{array}$ & $\begin{array}{c}-1.10 \\
(-1.093 /-1.107)\end{array}$ & $\begin{array}{c}721.50 \\
(716.00 / 726.00)\end{array}$ & $\begin{array}{c}0.10 \\
(0.099 / 0.101)\end{array}$ & & \\
\hline & $X N-E$ & $\begin{array}{c}494.00 \\
(466.00 / 506.00)\end{array}$ & $\begin{array}{c}-1.75 \\
(-1.651 /-1.793)\end{array}$ & 910 & $\begin{array}{c}502.00 \\
(502.00 / 508.00)\end{array}$ & $\begin{array}{c}-1.68 \\
(-1.670 /-1.693)\end{array}$ & $\begin{array}{c}725.00 \\
(720.00 / 730.00)\end{array}$ & $\begin{array}{c}0.60 \\
(0.596 / 0.604)\end{array}$ & & \\
\hline & OVM & $\begin{array}{c}502.80 \\
(481.80 / 518.80)\end{array}$ & & & $\begin{array}{c}510.60 \\
(507.40 / 513.40)\end{array}$ & & $\begin{array}{c}705.20 \\
(713.40 / 724.60)\end{array}$ & & & \\
\hline \multirow{6}{*}{$\begin{array}{l}\text { LY-WY } \\
\text { (ch) }\end{array}$} & $X N-A$ & $\begin{array}{c}844.00 \\
(806.00 / 871.00)\end{array}$ & $\begin{array}{c}1.01 \\
(0.965 / 1.042)\end{array}$ & 1426 & $\begin{array}{c}851.00 \\
(847.00 / 855.00)\end{array}$ & $\begin{array}{c}1.01 \\
(1.005 / 1.015)\end{array}$ & $\begin{array}{c}699.00 \\
(688.00 / 719.00)\end{array}$ & $\begin{array}{c}1.04 \\
(1.024 / 1.070)\end{array}$ & \multirow{6}{*}{$\begin{array}{c}1.30 \\
(0.80 / 1.90)\end{array}$} & \multirow{6}{*}{$\begin{array}{c}0.66 \\
(0.00 / 2.97)\end{array}$} \\
\hline & $X N-B$ & $\begin{array}{c}867.00 \\
(827.00 / 883.00)\end{array}$ & $\begin{array}{c}0.98 \\
(0.935 / 0.998)\end{array}$ & 1555 & $\begin{array}{c}865.00 \\
(859.00 / 868.00)\end{array}$ & $\begin{array}{c}1.00 \\
(0.993 / 1.003)\end{array}$ & $\begin{array}{c}751.50 \\
(721.00 / 758.00)\end{array}$ & $\begin{array}{c}0.97 \\
(0.931 / 0.978)\end{array}$ & & \\
\hline & $X N-C$ & $\begin{array}{c}845.00 \\
(816.00 / 874.00)\end{array}$ & $\begin{array}{c}1.01 \\
(0.975 / 1.045) \\
\end{array}$ & 1687 & $\begin{array}{c}863.00 \\
(860.00 / 867.00)\end{array}$ & $\begin{array}{c}1.00 \\
(0.997 / 1.005)\end{array}$ & $\begin{array}{c}720.00 \\
(713.00 / 754.00)\end{array}$ & $\begin{array}{c}1.01 \\
(1.000 / 1.058)\end{array}$ & & \\
\hline & XN-D & $\begin{array}{c}855.00 \\
(838.00 / 878.00)\end{array}$ & $\begin{array}{c}1.00 \\
(0.980 / 1.027)\end{array}$ & 1124 & $\begin{array}{c}878.00 \\
(872.00 / 883.00)\end{array}$ & $\begin{array}{c}0.98 \\
(0.973 / 0.986)\end{array}$ & $\begin{array}{c}750.50 \\
(739.00 / 768.00)\end{array}$ & $\begin{array}{c}0.97 \\
(0.955 / 0.993)\end{array}$ & & \\
\hline & $X N-E$ & $\begin{array}{c}857.00 \\
(829.00 / 879.00)\end{array}$ & $\begin{array}{c}1.00 \\
(0.967 / 1.026)\end{array}$ & 910 & $\begin{array}{c}857.00 \\
(851.00 / 861.00) \\
\end{array}$ & $\begin{array}{c}1.01 \\
(1.003 / 1.015)\end{array}$ & $\begin{array}{c}715.00 \\
(710.00 / 744.00)\end{array}$ & $\begin{array}{c}1.02 \\
(1.013 / 1.061)\end{array}$ & & \\
\hline & OVM & $\begin{array}{c}853.60 \\
(823.20 / 877.00)\end{array}$ & & & $\begin{array}{c}862.80 \\
(857.80 / 866.80)\end{array}$ & & $\begin{array}{c}727.20 \\
(714.20 / 748.60)\end{array}$ & & & \\
\hline \multirow{6}{*}{$\begin{array}{c}\text { LY-WZ } \\
\text { (ch) } \\
\S ¥\end{array}$} & $X N-A$ & $\begin{array}{c}691.00 \\
(679.00 / 715.00)\end{array}$ & $\begin{array}{c}14.06 \\
(13.816 / 14.548)\end{array}$ & 1426 & $\begin{array}{c}705.00 \\
(704.00 / 708.00)\end{array}$ & $\begin{array}{c}13.16 \\
(13.141 / 13.216)\end{array}$ & $\begin{array}{c}741.00 \\
(726.00 / 751.00)\end{array}$ & $\begin{array}{c}3.88 \\
(3.801 / 3.932)\end{array}$ & \multirow{6}{*}{$\begin{array}{c}0.70 \\
(0.50 / 1.10)\end{array}$} & \multirow{6}{*}{$\begin{array}{c}0.87 \\
(0.64 / 1.12)\end{array}$} \\
\hline & $X N-B$ & $\begin{array}{c}612.50 \\
(605.00 / 621.00)\end{array}$ & $\begin{array}{c}1.10 \\
(1.087 / 1.115)\end{array}$ & 1555 & $\begin{array}{c}632.00 \\
(630.00 / 633.00)\end{array}$ & $\begin{array}{c}1.44 \\
(1.435 / 1.442)\end{array}$ & $\begin{array}{c}715.50 \\
(704.00 / 723.00)\end{array}$ & $\begin{array}{c}0.76 \\
(0.748 / 0.768)\end{array}$ & & \\
\hline & $X N-C$ & $\begin{array}{c}595.00 \\
(586.00 / 608.00)\end{array}$ & $\begin{array}{c}-1.78 \\
(-1.753 /-1.819)\end{array}$ & 1687 & $\begin{array}{c}594.0 \\
(592.00 / 595.00)\end{array}$ & $\begin{array}{c}-4.65 \\
(-4.634 /-4.658)\end{array}$ & $\begin{array}{c}680.00 \\
(669.00 / 692.00)\end{array}$ & $\begin{array}{c}-3.24 \\
(-3.188 /-3.297)\end{array}$ & & \\
\hline & XN-D & $\begin{array}{c}511.50 \\
(502.00 / 528.00)\end{array}$ & $\begin{array}{c}-15.56 \\
(-15.271 /-16.062)\end{array}$ & 1124 & $\begin{array}{c}540.00 \\
(538.00 / 543.00)\end{array}$ & $(-13.271 /-13.394)$ & $\begin{array}{c}657.00 \\
(650.00 / 668.00)\end{array}$ & $\begin{array}{c}-7.16 \\
(-7.084 /-7.280)\end{array}$ & & \\
\hline & $X N-E$ & $\begin{array}{c}619.00 \\
(605.00 / 630.00)\end{array}$ & $\begin{array}{c}2.18 \\
(2.131 / 2.219)\end{array}$ & 910 & $\begin{array}{c}644.00 \\
(642.00 / 647.00)\end{array}$ & $\begin{array}{c}3.37 \\
(3.360 / 3.386)\end{array}$ & $\begin{array}{c}749.00 \\
(739.00 / 761.00)\end{array}$ & $\begin{array}{c}5.76 \\
(5.683 / 5.852)\end{array}$ & & \\
\hline & OVM & $\begin{array}{c}605.80 \\
(595.40 / 620.40)\end{array}$ & & & $\begin{array}{c}628.33 \\
(621.20 / 625.20)\end{array}$ & & $\begin{array}{c}681.73 \\
(697.60 / 719.00)\end{array}$ & & & \\
\hline
\end{tabular}

§: comparison of mean value of CPD parameter evaluated for all $5 \mathrm{XN}$ showed a significant difference in all pair comparisons by Kruskal-Wallis test with $p<0.0001$ in all groups of samples; $¥$ : comparison of mean value of CPD parameter evaluated for all $5 \mathrm{XN}$ showed a significative difference on ANOVA with $p<0.0001$ in all groups of samples. Highlighted in grey: Bias\% is lower than the $B_{A L G} \%$. B $B_{A L G}$ : alignment bias based on intra-individual biological variation; $B_{A P S}$ : analytical performance specification for Bias; LY-WX: lymphocyte complexity and width of dispersion of the events measured; LY-WY: lymphocyte fluorescence intensity and the width of dispersion; LY-WZ: lymphocyte cell size and the width of dispersion; LY-X: lymphocyte cell complexity; LY-Y: lymphocyte fluorescence intensity; LY-Z: lymphocyte cell size; OMV: overall median. 
Table IV Analyzers harmonization: monocytes cell population data. The median value and relative Bias (\%) (calculated in respect to overall median $[\mathrm{OMV}]$ ) of monocytes cell population data parameters to $\mathrm{B}_{\mathrm{APS}} \%(14)$ and target alignment Bias proposed by Fraser et al. $(0.33 \times \mathrm{CVI})\left(\mathrm{B}_{\mathrm{ALG}} \%\right)(15)$.

\begin{tabular}{|c|c|c|c|c|c|c|c|c|c|c|}
\hline & \multicolumn{3}{|c|}{$\begin{array}{l}30 \text { healthy blood donors } \\
\text { November } 2015\end{array}$} & \multicolumn{2}{|c|}{$\begin{array}{l}\text { Moving Average } \\
\text { November } 2015\end{array}$} & \multicolumn{2}{|c|}{$\begin{array}{l}\text { XN-Check (Normal) } \\
\text { November } 2015\end{array}$} & \multirow{2}{*}{$\begin{array}{l}B_{\text {APS }} \% \\
(95 \% \mathrm{Cl})\end{array}$} & \multirow{2}{*}{$\begin{array}{l}\mathrm{B}_{\mathrm{ALG}} \% \\
(95 \% \mathrm{Cl})\end{array}$} \\
\hline & & \begin{tabular}{l|} 
Median \\
$(95 \% \mathrm{Cl})$
\end{tabular} & $\begin{array}{c}\text { Bias\% vs OVM } \\
(95 \% \mathrm{Cl})\end{array}$ & $\begin{array}{c}\mathrm{N} \\
\text { samples }\end{array}$ & \begin{tabular}{l|} 
Median \\
$(95 \% \mathrm{Cl})$
\end{tabular} & $\begin{array}{c}\text { Bias\% vs OVM } \\
(95 \% \mathrm{Cl})\end{array}$ & $\begin{array}{l}\text { Median } \\
(95 \% \mathrm{Cl})\end{array}$ & $\begin{array}{c}\text { Bias\% vs OVM } \\
(95 \% \mathrm{Cl})\end{array}$ & & \\
\hline \multirow{6}{*}{$\begin{array}{c}\text { MO-X } \\
\text { (ch) } \\
\S ¥\end{array}$} & $\mathrm{XN}-\mathrm{A}$ & $\begin{array}{c}118.90 \\
(118.3 / 119.4)\end{array}$ & $\begin{array}{c}2.26 \\
(2.249 / 2.270)\end{array}$ & 1426 & $\begin{array}{c}119.20 \\
(119.10 / 119.30)\end{array}$ & $\begin{array}{c}2.09 \\
(2.088 / 2.092)\end{array}$ & $\begin{array}{c}129.15 \\
(127.80 / 130.80)\end{array}$ & $\begin{array}{c}-0.02 \\
(-0.020 /-0.020)\end{array}$ & \multirow{6}{*}{$\begin{array}{c}0.40 \\
(0.30 / 0.50)\end{array}$} & \multirow{6}{*}{$\begin{array}{c}0.36 \\
(0.30 / 0.46)\end{array}$} \\
\hline & $\mathrm{XN}-\mathrm{B}$ & $\begin{array}{c}115.45 \\
(114.7 / 115.7) \\
\end{array}$ & $\begin{array}{c}-0.71 \\
(-0.705 /-0.712)\end{array}$ & 1555 & \begin{tabular}{c|}
116.00 \\
$(115.90 / 116.10)$ \\
\end{tabular} & $\begin{array}{c}-0.65 \\
(-0.649 /-0.651)\end{array}$ & $\begin{array}{c}129.30 \\
(128.90 / 130.40)\end{array}$ & $\begin{array}{c}0.09 \\
(0.090 / 0.091)\end{array}$ & & \\
\hline & $\mathrm{XN}-\mathrm{C}$ & $\begin{array}{c}115.65 \\
(115.0 / 116.3) \\
\end{array}$ & $\begin{array}{c}-0.53 \\
(-0.527 /-0.533)\end{array}$ & 1687 & $\begin{array}{c}116.60 \\
(116.50 / 116.70) \\
\end{array}$ & $\begin{array}{c}-0.14 \\
(-0.139 /-0.140) \\
\end{array}$ & $\begin{array}{c}129.20 \\
(128.80 / 129.90) \\
\end{array}$ & $\begin{array}{c}0.02 \\
(0.019 / 0.020) \\
\end{array}$ & & \\
\hline & XN-D & $\begin{array}{c}115.45 \\
(114.90 / 116.00) \\
\end{array}$ & $\begin{array}{c}-0.71 \\
(-0.707 /-0.713)\end{array}$ & 1124 & $\begin{array}{c}115.90 \\
(115.80 / 116.00) \\
\end{array}$ & $\begin{array}{c}-0.74 \\
(-0.739 /-0.741) \\
\end{array}$ & $\begin{array}{c}129.55 \\
(128.90 / 130.00) \\
\end{array}$ & $\begin{array}{c}0.29 \\
(0.289 / 0.291) \\
\end{array}$ & & \\
\hline & $X N-E$ & $\begin{array}{c}115.90 \\
(114.90 / 116.30) \\
\end{array}$ & $\begin{array}{c}-0.32 \\
(-0.317 /-0.321)\end{array}$ & 910 & $\begin{array}{c}116.10 \\
(116.00 / 116.20 \\
\end{array}$ & $\begin{array}{c}-0.57 \\
(-0.569 /-0.570)\end{array}$ & $\begin{array}{c}128.70 \\
(128.40 / 129.70) \\
\end{array}$ & $\begin{array}{c}-0.37 \\
(-0.369 /-0.373)\end{array}$ & & \\
\hline & OVM & $\begin{array}{c}116.27 \\
(115.60 / 116.70)\end{array}$ & & & $\begin{array}{c}116.76 \\
(116.66 / 116.86)\end{array}$ & & $\begin{array}{c}129.18 \\
(128.56 / 130.16) \\
\end{array}$ & & & \\
\hline \multirow{6}{*}{$\begin{array}{c}\text { MO-Y } \\
\text { (ch) } \\
\$ ¥\end{array}$} & $\mathrm{XN}-\mathrm{A}$ & $\begin{array}{c}113.25 \\
(112.10 / 116.30)\end{array}$ & $\begin{array}{c}1.13 \\
(1.119 / 1.160)\end{array}$ & 1426 & $\begin{array}{c}115.20 \\
(114.80 / 115.60)\end{array}$ & $\begin{array}{c}1.53 \\
(1.525 / 1.535)\end{array}$ & $\begin{array}{c}124.30 \\
(121.80 / 126.00)\end{array}$ & $\begin{array}{c}-0.15 \\
(-0.147 /-0.152)\end{array}$ & \multirow{6}{*}{$\begin{array}{c}0.80 \\
(0.60 / 1.20)\end{array}$} & \multirow{6}{*}{$\begin{array}{c}0.86 \\
(0.66 / 1.09)\end{array}$} \\
\hline & $\mathrm{XN}-\mathrm{B}$ & $\begin{array}{c}110.05 \\
(108.40 / 111.50)\end{array}$ & $\begin{array}{c}-1.72 \\
(-1.694 /-1.743)\end{array}$ & 1555 & \begin{tabular}{c|}
112.00 \\
$(111.60 / 112.30)$ \\
\end{tabular} & $\begin{array}{c}-1.29 \\
(-1.285 /-1.293)\end{array}$ & \begin{tabular}{c|}
120.70 \\
$(120.20 / 122.50)$ \\
\end{tabular} & $\begin{array}{c}-3.04 \\
(-3.027 /-3.085)\end{array}$ & & \\
\hline & $\mathrm{XN}-\mathrm{C}$ & $\begin{array}{c}114.95 \\
(113.60 / 117.20) \\
\end{array}$ & $\begin{array}{c}2.65 \\
(2.619 / 2.702) \\
\end{array}$ & 1687 & $\begin{array}{c}118.30 \\
(118.00 / 118.60) \\
\end{array}$ & $\begin{array}{c}4.27 \\
(4.259 / 4.281) \\
\end{array}$ & $\begin{array}{c}129.50 \\
(129.00 / 131.50) \\
\end{array}$ & $\begin{array}{c}4.02 \\
(4.004 / 4.082) \\
\end{array}$ & & \\
\hline & XN-D & $\begin{array}{c}108.50 \\
(106.50 / 112.20)\end{array}$ & $\begin{array}{c}-3.11 \\
(-3.053 /-3.216)\end{array}$ & 1124 & $\begin{array}{c}109.70 \\
(109.20 / 110.10)\end{array}$ & $\begin{array}{c}-3.31 \\
(-3.295 /-3.322)\end{array}$ & $\begin{array}{c}123.65 \\
(123.00 / 125.00) \\
\end{array}$ & $\begin{array}{c}-0.67 \\
(-0.666 /-0.677)\end{array}$ & & \\
\hline & $\mathrm{XN}-\mathrm{E}$ & $\begin{array}{c}113.15 \\
(110.70 / 115.30) \\
\end{array}$ & $\begin{array}{c}1.04 \\
(1.017 / 1.060)\end{array}$ & 910 & $\begin{array}{c}112.10 \\
(111.80 / 112.40) \\
\end{array}$ & $\begin{array}{c}-1.20 \\
(-1.197 /-1.203)\end{array}$ & $\begin{array}{c}124.30 \\
(123.80 / 125.00)\end{array}$ & $\begin{array}{c}-0.15 \\
(-0.149 /-0.151)\end{array}$ & & \\
\hline & OVM & $\begin{array}{c}111.98 \\
(110.30 / 114.50) \\
\end{array}$ & & & \begin{tabular}{|c|}
113.46 \\
$(113.08 / 113.80)$ \\
\end{tabular} & & \begin{tabular}{|c|}
124.49 \\
$(123.56 / 126.00)$ \\
\end{tabular} & & & \\
\hline \multirow{6}{*}{$\begin{array}{c}\text { MO-Z } \\
\text { (ch) } \\
\S ¥\end{array}$} & $\mathrm{XN}-\mathrm{A}$ & $\begin{array}{c}68.50 \\
(67.40 / 69.10)\end{array}$ & $\begin{array}{c}4.13 \\
(4.064 / 4.166)\end{array}$ & 1426 & $\begin{array}{c}68.50 \\
(68.30 / 68.60)\end{array}$ & $\begin{array}{c}4.93 \\
(4.916 / 4.937)\end{array}$ & $\begin{array}{c}105.00 \\
(103.50 / 106.20) \\
\end{array}$ & $\begin{array}{c}-0.58 \\
(-0.572 /-0.587)\end{array}$ & \multirow{6}{*}{$\begin{array}{c}0.50 \\
(0.30 / 0.70)\end{array}$} & \multirow{6}{*}{$\begin{array}{c}0.33 \\
(0.00 / 0.53)\end{array}$} \\
\hline & $\mathrm{XN}-\mathrm{B}$ & $\begin{array}{c}64.90 \\
(64.50 / 65.20)\end{array}$ & $\begin{array}{c}-1.34 \\
(-1.332 /-1.346)\end{array}$ & 1555 & $\begin{array}{c}64.60 \\
(64.50 / 64.80)\end{array}$ & $\begin{array}{c}-1.04 \\
(-1.038 /-1.043)\end{array}$ & $\begin{array}{c}104.25 \\
(103.30 / 105.30) \\
\end{array}$ & $\begin{array}{c}-1.29 \\
(-1.278 /-1.303)\end{array}$ & & \\
\hline & $\mathrm{XN}-\mathrm{C}$ & $\begin{array}{c}63.80 \\
(63.00 / 64.50)\end{array}$ & $\begin{array}{c}-3.01 \\
(-2.972 /-3.043)\end{array}$ & 1687 & $\begin{array}{c}64.30 \\
(64.20 / 64.40)\end{array}$ & $\begin{array}{c}-1.50 \\
(-1.498 /-1.502)\end{array}$ & \begin{tabular}{|c|}
105.10 \\
$(103.30 / 105.30)$ \\
\end{tabular} & $\begin{array}{c}-0.48 \\
(-0.475 /-0.485)\end{array}$ & & \\
\hline & XN-D & $\begin{array}{c}63.40 \\
(63.20 / 64.10)\end{array}$ & $\begin{array}{c}-3.62 \\
(-3.609 /-3.660)\end{array}$ & 1124 & $\begin{array}{c}62.60 \\
(62.50 / 62.70)\end{array}$ & $\begin{array}{c}-4.11 \\
(-4.103 /-4.117)\end{array}$ & $\begin{array}{c}107.10 \\
(106.60 / 107.80) \\
\end{array}$ & $\begin{array}{c}1.41 \\
(1.403 / 1.419)\end{array}$ & & \\
\hline & $X N-E$ & $\begin{array}{c}68.30 \\
(67.80 / 68.80)\end{array}$ & $\begin{array}{c}3.83 \\
(3.802 / 3.858)\end{array}$ & 910 & $\begin{array}{c}66.40 \\
(66.20 / 66.60)\end{array}$ & $\begin{array}{c}1.72 \\
(1.715 / 1.725)\end{array}$ & $\begin{array}{c}106.60 \\
(104.80 / 107.60) \\
\end{array}$ & $\begin{array}{c}0.94 \\
(0.924 / 0.949)\end{array}$ & & \\
\hline & OVM & $\begin{array}{c}65.78 \\
(65.18 / 66.34)\end{array}$ & & & $\begin{array}{c}65.28 \\
(65.14 / 65.42)\end{array}$ & & $\begin{array}{c}105.61 \\
(104.46 / 106.64)\end{array}$ & & & \\
\hline \multirow{6}{*}{$\begin{array}{c}\text { MO-WX } \\
\text { (ch) } \\
\S ¥\end{array}$} & $\mathrm{XN}-\mathrm{A}$ & $\begin{array}{c}264.50 \\
(258.00 / 274.00)\end{array}$ & $\begin{array}{c}5.00 \\
(4.877 / 5.180)\end{array}$ & 1426 & \begin{tabular}{c|}
263.00 \\
$(262.00 / 265.00)$ \\
\end{tabular} & $\begin{array}{c}3.46 \\
(3.447 / 3.486)\end{array}$ & $\begin{array}{c}383.00 \\
(379.00 / 391.00)\end{array}$ & $\begin{array}{c}4.41 \\
(4.364 / 4.502)\end{array}$ & \multirow{6}{*}{$\begin{array}{c}1.10 \\
(0.50 / 1.70)\end{array}$} & \multirow{6}{*}{$\begin{array}{c}1.39 \\
(0.73 / 1.94)\end{array}$} \\
\hline & $\mathrm{XN}-\mathrm{B}$ & $\begin{array}{c}247.00 \\
(241.00 / 258.00)\end{array}$ & $\begin{array}{c}-1.94 \\
(-1.893 /-2.026)\end{array}$ & 1555 & $\begin{array}{c}251.00 \\
(250.00 / 252.00) \\
\end{array}$ & $\begin{array}{c}-1.26 \\
(-1.255 /-1.265)\end{array}$ & $\begin{array}{c}359.50 \\
(356.00 / 362.00) \\
\end{array}$ & $\begin{array}{c}-0.71 \\
(-0.703 /-0.715)\end{array}$ & & \\
\hline & $\mathrm{XN}-\mathrm{C}$ & $\begin{array}{c}248.00 \\
(242.00 / 251.00)\end{array}$ & $\begin{array}{c}-1.55 \\
(-1.513 /-1.569)\end{array}$ & 1687 & $\begin{array}{c}253.00 \\
(252.00 / 255.00)\end{array}$ & $\begin{array}{c}-0.47 \\
(-0.468 /-0.474)\end{array}$ & $\begin{array}{c}375.00 \\
(368.00 / 379.00) \\
\end{array}$ & $\begin{array}{c}2.11 \\
(2.071 / 2.133) \\
\end{array}$ & & \\
\hline & $X N-D$ & $\begin{array}{c}250.50 \\
(244.00 / 255.00)\end{array}$ & $\begin{array}{c}-0.56 \\
(-0.545 /-0.570)\end{array}$ & 1124 & $\begin{array}{c}254.00 \\
(253.00 / 256.00) \\
\end{array}$ & $\begin{array}{c}-0.08 \\
(-0.079 /-0.081\end{array}$ & $\begin{array}{c}361.00 \\
(355.00 / 364.00) \\
\end{array}$ & $\begin{array}{c}-3.25 \\
(-3.196 /-3.277)\end{array}$ & & \\
\hline & $\mathrm{XN}-\mathrm{E}$ & $\begin{array}{c}249.50 \\
(241.00 / 258.00)\end{array}$ & $\begin{array}{c}-0.95 \\
(-0.918 /-0.982)\end{array}$ & 910 & $\begin{array}{c}250.00 \\
(249.00 / 252.00)\end{array}$ & $\begin{array}{c}-1.65 \\
(-1.643 /-1.663)\end{array}$ & $\begin{array}{c}365.50 \\
(360.00 / 370.00)\end{array}$ & $\begin{array}{c}-2.57 \\
(-2.531 /-2.602)\end{array}$ & & \\
\hline & OVM & $\begin{array}{c}251.90 \\
(245.20 / 259.20)\end{array}$ & & & \begin{tabular}{|c|}
254.20 \\
$(253.20 / 256.00)$
\end{tabular} & & \begin{tabular}{|c|}
354.52 \\
$(363.60 / 373.20)$ \\
\end{tabular} & & & \\
\hline \multirow{6}{*}{$\begin{array}{c}\text { MO-WY } \\
\text { (ch) }\end{array}$} & $\mathrm{XN}-\mathrm{A}$ & $\begin{array}{c}6777.00 \\
(643.00 / 690.00)\end{array}$ & $\begin{array}{c}0.99 \\
(0.940 / 1.009)\end{array}$ & 1426 & $\begin{array}{c}674.00 \\
(667.00 / 678.00) \\
\end{array}$ & $\begin{array}{c}1.00 \\
(0.990 / 1.006)\end{array}$ & $\begin{array}{c}873.00 \\
(844.00 / 905.00)\end{array}$ & $\begin{array}{c}0.96 \\
(0.928 / 0.995)\end{array}$ & \multirow{6}{*}{$\begin{array}{c}1.60 \\
(0.70 / 2.60)\end{array}$} & \multirow{6}{*}{$\begin{array}{c}1.60 \\
(0.54 / 2.38)\end{array}$} \\
\hline & $\mathrm{XN}-\mathrm{B}$ & $\begin{array}{c}636.50 \\
(611.00 / 692.00)\end{array}$ & $\begin{array}{c}1.05 \\
(1.008 / 1.142)\end{array}$ & 1555 & $\begin{array}{c}672.00 \\
(668.00 / 677.00)\end{array}$ & $\begin{array}{c}1.00 \\
(0.994 / 1.007)\end{array}$ & $\begin{array}{c}818.00 \\
(792.00 / 867.00)\end{array}$ & $\begin{array}{c}1.03 \\
(0.997 / 1.092)\end{array}$ & & \\
\hline & $\mathrm{XN}-\mathrm{C}$ & $\begin{array}{c}665.00 \\
(637.00 / 715.00)\end{array}$ & $\begin{array}{c}1.01 \\
(1.008 / 1.142)\end{array}$ & 1687 & $\begin{array}{c}666.00 \\
(662.00 / 673.00)\end{array}$ & $\begin{array}{c}1.01 \\
(1.004 / 1.021)\end{array}$ & $\begin{array}{c}859.00 \\
(824.00 / 882.00)\end{array}$ & $\begin{array}{c}0.98 \\
(0.940 / 1.006)\end{array}$ & & \\
\hline & XN-D & $\begin{array}{c}690.50 \\
(636.00 / 761.00)\end{array}$ & $\begin{array}{c}0.97 \\
(0.967 / 1.086)\end{array}$ & 1124 & $\begin{array}{c}674.00 \\
(668.00 / 681.00)\end{array}$ & $\begin{array}{c}1.00 \\
(0.991 / 1.010)\end{array}$ & $\begin{array}{c}857.50 \\
(806.00 / 859.00)\end{array}$ & $\begin{array}{c}0.98 \\
(0.921 / 0.982)\end{array}$ & & \\
\hline & $\mathrm{XN}-\mathrm{E}$ & $\begin{array}{c}677.50 \\
(639.00 / 708.00)\end{array}$ & $\begin{array}{c}0.99 \\
(0.893 / 1.069)\end{array}$ & 910 & $\begin{array}{c}677.00 \\
(670.00 / 683.00)\end{array}$ & $\begin{array}{c}0.99 \\
(0.980 / 0.999)\end{array}$ & $\begin{array}{c}800.00 \\
(800.00 / 839.00)\end{array}$ & $\begin{array}{c}1.05 \\
(1.050 / 1.101)\end{array}$ & & \\
\hline & OVM & $\begin{array}{c}669.30 \\
(633.20 / 713.20)\end{array}$ & & & $\begin{array}{c}672.60 \\
(667.00 / 678.40)\end{array}$ & & $\begin{array}{c}841.50 \\
(813.20 / 870.40)\end{array}$ & & & \\
\hline \multirow{6}{*}{$\begin{array}{c}\text { MO-WZ } \\
\text { (ch) } \\
\S ¥\end{array}$} & $\mathrm{XN}-\mathrm{A}$ & $\begin{array}{c}789.00 \\
(750.0 / 818.00)\end{array}$ & $\begin{array}{c}12.28 \\
(11.673 / 12.731\end{array}$ & 1426 & \begin{tabular}{c|}
779.00 \\
$(774.00 / 782.00)$
\end{tabular} & $\begin{array}{c}10.87 \\
(10.800 / 10.912)\end{array}$ & $\begin{array}{c}880.00 \\
(858.00 / 894.00)\end{array}$ & $\begin{array}{c}4.82 \\
(4.700 / 4.897)\end{array}$ & \multirow{6}{*}{$\begin{array}{c}1.10 \\
(0.70 / 1.80)\end{array}$} & \multirow{6}{*}{$\begin{array}{c}1.06 \\
(0.00 / 1.60)\end{array}$} \\
\hline & $\mathrm{XN}-\mathrm{B}$ & $\begin{array}{c}683.00 \\
(667.00 / 708.00)\end{array}$ & $\begin{array}{c}-2.80 \\
(-2.734 /-2.902)\end{array}$ & 1555 & \begin{tabular}{|c|}
697.00 \\
$(694.00 / 699.00)$
\end{tabular} & $\begin{array}{c}-0.80 \\
(-0.797 /-0.802)\end{array}$ & $\begin{array}{c}813.50 \\
(794.00 / 840.00)\end{array}$ & $\begin{array}{c}-3.66 \\
(-3.572 /-3.779)\end{array}$ & & \\
\hline & $\mathrm{XN}-\mathrm{C}$ & $\begin{array}{c}653.50 \\
(625.00 / 669.00)\end{array}$ & $\begin{array}{c}-7.00 \\
(-6.695 /-7.166)\end{array}$ & 1687 & $\begin{array}{c}643.00 \\
(639.00 / 646.00)\end{array}$ & $\begin{array}{c}-8.48 \\
(-8.427 /-8.520)\end{array}$ & $\begin{array}{c}792.00 \\
(776.00 / 806.00)\end{array}$ & $\begin{array}{c}-4.02 \\
(-3.939 /-4.091)\end{array}$ & & \\
\hline & XN-D & $\begin{array}{c}625.00 \\
(611.00 / 650.00) \\
\end{array}$ & $\begin{array}{c}-11.06 \\
(-10.812 /-11.502)\end{array}$ & 1124 & $\begin{array}{c}624.00 \\
(620.00 / 630.00) \\
\end{array}$ & $\begin{array}{c}-11.19 \\
(-11.118 /-11.298)\end{array}$ & $\begin{array}{c}773.00 \\
(765.00 / 789.00) \\
\end{array}$ & $\begin{array}{c}-8.48 \\
(-8.392 /-8.656)\end{array}$ & & \\
\hline & $X N-E$ & $\begin{array}{c}763.00 \\
(743.00 / 793.00)\end{array}$ & $\begin{array}{c}8.58 \\
(8.355 / 8.917) \\
\end{array}$ & 910 & \begin{tabular}{c|}
770.00 \\
$(762.00 / 776.00)$
\end{tabular} & $\begin{array}{c}9.59 \\
(9.490 / 9.665)\end{array}$ & $\begin{array}{c}941.50 \\
(919.00 / 954.00)\end{array}$ & $\begin{array}{c}11.33 \\
(11.059 / 11.480)\end{array}$ & & \\
\hline & OVM & $\begin{array}{c}702.70 \\
(679.20 / 727.60)\end{array}$ & & & $\begin{array}{c}702.60 \\
(697.80 / 706.60) \\
\end{array}$ & & \begin{tabular}{|c|}
784.50 \\
$(822.40 / 856.60)$ \\
\end{tabular} & & & \\
\hline
\end{tabular}

$\S$ : comparison of mean value of CPD parameter evaluated for all $5 \mathrm{XN}$ showed a significant difference in all pair comparisons by Kruskal-Wallis test with $\mathrm{p}<0.0001$ in all groups of samples; $¥$ : comparison of mean value of CPD parameter evaluated for all $5 \mathrm{XN}$ showed a significant difference on ANOVA with $\mathrm{p}<0.0001$ in all groups of samples. Highlighted in grey: Bias\% is lower than the $B_{A L G} \%$. $B_{A L G}$ : alignment bias based on intra-individual biological variation; $B_{A P S}$ : analytical performance specification for Bias; MOWX: monocyte complexity and width of dispersion of the events measured; MO-WY: monocyte fluorescence intensity and the width of dispersion; MO-WZ: monocyte cell size and the width of dispersion; MO-X: monocyte cell complexity; MO-Y: monocyte fluorescence intensity; MO-Z: monocyte cell size; OMV: overall median 
Table V Stability of CPD parameters in normal blood samples. Median Hodges-Lehmann location shift $(\triangle \mathrm{X})$; Bias\% (B\%) between baseline (TO) and the time point ( $2 \mathrm{~h}$ up to $48 \mathrm{~h}$ ) at $4{ }^{\circ} \mathrm{C}$ and Room Temperature (RT) comparison to B APS \% and Reference Change Value \% (RCV\%).

\begin{tabular}{|c|c|c|c|c|c|c|c|c|c|c|c|c|}
\hline \multirow{2}{*}{ Parameters } & \multirow{2}{*}{$\mathrm{T}\left[{ }^{\circ} \mathrm{C}\right]$} & \multirow{2}{*}{$\begin{array}{l}\text { T0 median value } \\
(95 \% \mathrm{Cl})\end{array}$} & \multicolumn{7}{|c|}{$\begin{array}{c}\Delta \mathrm{X}(\mathrm{T}-\mathrm{TO}) \text { absolute value } \\
\text { Hodges-Lehmann location shift }\end{array}$} & \multirow{2}{*}{$\begin{array}{c}\Delta \mathrm{X} \text { not } \\
\text { significant } \\
\text { until }[\mathrm{h}]\end{array}$} & \multirow{2}{*}{$\begin{array}{l}\mathrm{B} \%(95 \% \mathrm{Cl}) \\
\text { at time of stability; } \\
\text { stable until }[\mathrm{h}]\end{array}$} & \multirow{2}{*}{$\begin{array}{c}\mathrm{B} \%(95 \% \mathrm{Cl}) \text { at } \\
\text { time is equal or lower } \\
\text { than } \mathrm{RCV} \% \text {; no } \\
\text { clinical impact until } \mathrm{h}\end{array}$} \\
\hline & & & $2 \mathrm{~h}$ & $4 \mathrm{~h}$ & $6 \mathrm{~h}$ & $8 \mathrm{~h}$ & $24 \mathrm{~h}$ & $36 \mathrm{~h}$ & $48 \mathrm{~h}$ & & & \\
\hline \multirow{2}{*}{$\begin{array}{l}\text { NE-SSC } \\
\text { (ch) }\end{array}$} & RT & \multirow{2}{*}{$\begin{array}{c}147.8 \\
(144.4 \text { to } 152.2)\end{array}$} & -0.53 & -1.19 & -1.63 & -2.2 & -7.37 & -10.7 & -14.8 & $8 \mathrm{~h}$ & $\begin{array}{c}-0.4(-0.6 \text { to- } 0.1) ; \\
2 \mathrm{~h}\end{array}$ & $\begin{array}{l}-1.5(-1.8 \text { to }-1.1) ; \\
8 \mathrm{~h}\end{array}$ \\
\hline & $4^{\circ} \mathrm{C}$ & & -1.85 & -0.65 & -2.1 & -1.15 & -3.95 & -4.5 & -6.6 & $36 \mathrm{~h}$ & not stable $\mathrm{p}<0.0001$ & $\begin{array}{l}-0.5(-0.9 \text { to }-0.0) ; \\
8 h\end{array}$ \\
\hline \multirow{2}{*}{$\begin{array}{l}\text { NE-SFL } \\
\text { (ch) }\end{array}$} & RT & \multirow{2}{*}{$\begin{array}{c}46.9 \\
(46.2 \text { to } 50.2)\end{array}$} & 0.3 & 0.15 & -0.8 & -0.6 & -3.1 & -3.75 & -3.7 & $8 \mathrm{~h}$ & $\begin{array}{c}0.3(-1.1 \text { to } 1.7) ; \\
4 \mathrm{~h}\end{array}$ & $\begin{array}{c}-7.2(-9.0 \text { to }-5.4) ; \\
24 h\end{array}$ \\
\hline & $4^{\circ} \mathrm{C}$ & & -0.55 & -0.3 & -1.3 & 0.10 & 1.5 & 1.45 & 1.55 & $\begin{array}{c}\text { no } \\
\text { difference }\end{array}$ & $\begin{array}{c}0.5(-0.7 \text { to } 1.6) ; \\
8 \mathrm{~h}\end{array}$ & $\begin{array}{l}\text { no clinical impact } \\
\text { over } 48 \mathrm{~h}\end{array}$ \\
\hline \multirow{2}{*}{$\begin{array}{l}\text { NE-FSC } \\
\text { (ch) }\end{array}$} & RT & \multirow{2}{*}{$\begin{array}{c}84.7 \\
(83.4 \text { to } 87.5)\end{array}$} & 1.7 & 0.2 & 0.2 & -0.65 & -12.3 & -17.45 & -2.1 & $8 \mathrm{~h}$ & $\begin{array}{c}-0.7(-2.2 \text { to } 0.7) ; \\
8 \mathrm{~h}\end{array}$ & $\begin{array}{c}-0.7(-2.2 \text { to } 0.7) ; \\
24 \mathrm{~h}\end{array}$ \\
\hline & $4^{\circ} \mathrm{C}$ & & 4.4 & 4.6 & 4.4 & 6.2 & 3.8 & 3.2 & 1.5 & $\begin{array}{l}\text { not stable } \\
p<0.0001\end{array}$ & not stable $p<0.0001$ & $\begin{array}{c}\text { no clinical impact } \\
\text { over } 48 \mathrm{~h}\end{array}$ \\
\hline \multirow{2}{*}{$\begin{array}{l}\text { NE-WX } \\
\text { (ch) }\end{array}$} & RT & \multirow{2}{*}{$\begin{array}{c}312.5 \\
(291 \text { to } 328)\end{array}$} & 6.8 & 5.4 & 8.4 & 13.6 & 97.4 & 129.9 & 121.3 & $8 \mathrm{~h}$ & no stable $\mathrm{p}<0.0001$ & $\begin{array}{c}4.3(2.3 \text { to } 6.3) \\
8 \mathrm{~h}\end{array}$ \\
\hline & $4^{\circ} \mathrm{C}$ & & 23.0 & 28.0 & 28.0 & 31.0 & 44.0 & 55.5 & 77.5 & $\begin{array}{l}\text { not stable } \\
p<0.0001\end{array}$ & not stable $\mathrm{p}<0.0001$ & $\begin{array}{c}8.8(6.22 \text { to } 11.46) ; \\
8 \mathrm{~h}\end{array}$ \\
\hline \multirow{2}{*}{$\begin{array}{l}\text { NE-WY } \\
\text { (ch) }\end{array}$} & RT & \multirow{2}{*}{$\begin{array}{c}591.5 \\
(578 \text { to } 615)\end{array}$} & 0.20 & -3.20 & -0.35 & 9.15 & 252.1 & 356.6 & 251.95 & $8 \mathrm{~h}$ & $\begin{array}{c}-0.05(-1.6 \text { to } 1.5) ; \\
6 h\end{array}$ & $\begin{array}{c}1.5(-0.6 \text { to } 3.7) ; \\
8 \mathrm{~h}\end{array}$ \\
\hline & $4^{\circ} \mathrm{C}$ & & 9.5 & 4.0 & 17.5 & 3.0 & 34.0 & 31.0 & 71.0 & $8 \mathrm{~h}$ & not stable $\mathrm{p}<0.0001$ & $\begin{array}{c}5.5(2.1 \text { to } 8.9) ; \\
24 \mathrm{~h}\end{array}$ \\
\hline \multirow{2}{*}{$\begin{array}{l}\text { NE-WZ } \\
\text { (ch) }\end{array}$} & RT & \multirow{2}{*}{$\begin{array}{c}649.5 \\
(632 \text { to } 749)\end{array}$} & -9.0 & -7.0 & -3.0 & 19.0 & 524.5 & 756.5 & 425.5 & $8 \mathrm{~h}$ & $\begin{array}{c}2.5(-2.3 \text { to } 7.2) ; \\
8 \mathrm{~h}\end{array}$ & $\begin{array}{c}2.5(-2.3 \text { to } 7.2) \\
8 \mathrm{~h} \\
\end{array}$ \\
\hline & $4^{\circ} \mathrm{C}$ & & -21.0 & 15.0 & 13.0 & 39.0 & 52.0 & 76.5 & 140.0 & $24 \mathrm{~h}$ & $\begin{array}{c}0.6(-5.1 \text { to } 6.4) ; \\
6 \mathrm{~h}\end{array}$ & $\begin{array}{c}9.0(5.2 \text { to } 12.7) ; \\
24 \mathrm{~h}\end{array}$ \\
\hline \multirow{2}{*}{$\begin{array}{l}\text { LY-X } \\
\text { (ch) }\end{array}$} & RT & \multirow{2}{*}{$\begin{array}{c}77.7 \\
\text { (76.0 to } 78.2)\end{array}$} & 0.41 & 0.55 & 0.64 & 1.07 & 1.49 & 2.7 & 3.17 & $24 \mathrm{~h}$ & $\begin{array}{c}0.7(0.0 \text { to } 1.4) ; \\
4 \mathrm{~h}\end{array}$ & $\begin{array}{c}\text { no clinical impact } \\
\text { over } 48 \mathrm{~h}\end{array}$ \\
\hline & $4^{\circ} \mathrm{C}$ & & 1.4 & 2.0 & 1.75 & 2.5 & 2.3 & 2.8 & 2.75 & $6 \mathrm{~h}$ & not stable $p<0.0001$ & $\begin{array}{c}\text { no clinical impact } \\
\text { over } 48 \mathrm{~h}\end{array}$ \\
\hline \multirow{2}{*}{$\begin{array}{l}\text { LY-Y } \\
\text { (ch) }\end{array}$} & RT & \multirow{2}{*}{$\begin{array}{c}71.6 \\
(68.5 \text { to } 73.1)\end{array}$} & -0.02 & -0.09 & -0.82 & -1.0 & -5.04 & -4.83 & -7.28 & $8 \mathrm{~h}$ & $\begin{array}{c}-0.1(-1.7 \text { to } 1.5) ; \\
4 \mathrm{~h}\end{array}$ & $\begin{array}{c}-7.3(-8.9 \text { to }-5.7) ; \\
24 h\end{array}$ \\
\hline & $4^{\circ} \mathrm{C}$ & & 0.0 & 0.2 & -0.55 & 0.4 & -0.3 & -0.5 & -2.5 & $\begin{array}{c}\text { no } \\
\text { difference }\end{array}$ & $\begin{array}{c}-0.3(-1.3 \text { to } 0.8) ; \\
24 \mathrm{~h}\end{array}$ & $\begin{array}{c}\text { no clinical impact } \\
\text { over } 48 \mathrm{~h}\end{array}$ \\
\hline \multirow{2}{*}{$\begin{array}{l}\mathrm{LY}-\mathrm{Z} \\
\text { (ch) }\end{array}$} & RT & 55.1 & -0.1 & -0.4 & -1.0 & -0.9 & -0.7 & -1.1 & 0.1 & $\begin{array}{c}\text { no } \\
\text { difference } \\
\end{array}$ & $\begin{array}{c}0.05(-0.6 \text { to } 0.7) ; \\
2 \mathrm{~h}\end{array}$ & $\begin{array}{c}\text { no clinical impact } \\
\text { over } 48 \mathrm{~h}\end{array}$ \\
\hline & $4^{\circ} \mathrm{C}$ & (54.6 to 62.7 ) & 0.7 & 2.1 & 1.9 & 3.3 & 3.8 & 3.4 & 3.0 & $6 \mathrm{~h}$ & not stable $p<0.0001$ & $\begin{array}{c}\text { no clinical impact } \\
\text { over } 48 \mathrm{~h}\end{array}$ \\
\hline LY-WX & RT & 486.0 & 16.5 & 6.5 & 10.5 & 12.0 & 32.5 & 19.0 & 46.0 & $36 \mathrm{~h}$ & $\begin{array}{c}2.4(-1.5 \text { to } 6.2) ; \\
8 \mathrm{~h}\end{array}$ & $\begin{array}{l}\text { no clinical impact } \\
\text { over } 48 \mathrm{~h}\end{array}$ \\
\hline (ch) & $4^{\circ} \mathrm{C}$ & (460.0 to 502.0$)$ & -3.0 & 4.0 & -4.0 & -17.0 & 71.0 & 97.5 & 112.5 & $8 \mathrm{~h}$ & $\begin{array}{c}-1.0(-5.4 \text { to } 3.4) ; \\
6 \mathrm{~h}\end{array}$ & $\begin{array}{c}3.4(-7.4 \text { to } 0.5) ; \\
8 \mathrm{~h}\end{array}$ \\
\hline LY-WY & RT & 854.5 & 9.8 & 2.1 & 12.35 & 31.75 & 144.9 & 209.1 & 211.15 & $8 \mathrm{~h}$ & $\begin{array}{c}0.2(-4.2 \text { to } 4.6) ; \\
2 \mathrm{~h}\end{array}$ & $\begin{array}{c}3.8(0.5 \text { to } 7.1) ; \\
8 \mathrm{~h}\end{array}$ \\
\hline ( & $4^{\circ} \mathrm{C}$ & (808 to & -8.0 & -9.5 & 0.0 & 5.0 & 56.0 & 81.0 & 195.5 & $24 \mathrm{~h}$ & $\begin{array}{c}1.2(-3.4 \text { to } 5.7) ; \\
8 \mathrm{~h}\end{array}$ & $\begin{array}{c}1.2(-3.4 \text { to } 5.8) ; \\
8 \mathrm{~h}\end{array}$ \\
\hline LY-WZ & RT & & -13.4 & -27.85 & -9.6 & 5.5 & 132.1 & 196.0 & 307.5 & $8 \mathrm{~h}$ & $\begin{array}{c}0.96(1.7 \text { to } 3.6) ; \\
8 \mathrm{~h}\end{array}$ & $\begin{array}{c}-2.6(-6.1 \text { to } 0.9) ; \\
24 \mathrm{~h}\end{array}$ \\
\hline (ch) & $4^{\circ} \mathrm{C}$ & (527 to 578 ) & -24.0 & -23.0 & -15.0 & 3.0 & 118.5 & 118.0 & 277.0 & $\begin{array}{l}\text { not stable } \\
p<0.0001\end{array}$ & not stable $p<0.0001$ & not stable $p<0.0001$ \\
\hline MO-X & RT & 115.2 & 0.22 & 0.0 & -0.87 & -0.35 & -3.43 & -2.97 & -2.41 & $8 \mathrm{~h}$ & $\begin{array}{c}-0.1(-0.6 \text { to }-0.06) ; \\
4 \mathrm{~h} \\
\end{array}$ & $\begin{array}{c}\text { no clinical impact } \\
\text { over } 48 \mathrm{~h}\end{array}$ \\
\hline & $4^{\circ} \mathrm{C}$ & & 1.7 & 1.9 & 1.9 & 2.8 & 5.8 & 6.5 & 7.9 & $\begin{array}{l}\text { not stable } \\
p<0.0001\end{array}$ & not stable $p<0.0001$ & $\begin{array}{c}2.5(2.0 \text { to } 2.9) ; \\
8 \mathrm{~h}\end{array}$ \\
\hline MO-Y & RT & 109.3 & 1.36 & -0.13 & -2.4 & -1.7 & -16.3 & -21.4 & -28.3 & $8 \mathrm{~h}$ & $\begin{array}{c}-0.1(-1.7 \text { to } 1.4) ; \\
4 \mathrm{~h}\end{array}$ & $\begin{array}{c}-1.6(-3.4 \text { to } 0.3) ; \\
8 \mathrm{~h}\end{array}$ \\
\hline (ch) & $4^{\circ} \mathrm{C}$ & (106.2 to 111.3 ) & -3.3 & -1.6 & -5.4 & -2.55 & -0.35 & -0.65 & -4.45 & $4 \mathrm{~h}$ & not stable $\mathrm{p}<0.0001$ & $\begin{array}{l}\text { no clinical impact } \\
\text { over } 48 \mathrm{~h}\end{array}$ \\
\hline MO-Z & RT & 59.3 & -0.1 & -0.7 & -1.6 & -1.3 & -3.25 & -7.95 & -4.55 & $24 \mathrm{~h}$ & $\begin{array}{c}0.3(-1.1 \text { to } 1.8) ; \\
2 \mathrm{~h}\end{array}$ & $\begin{array}{c}-3.0(-5.3 \text { to }-0.7) ; \\
8 \mathrm{~h}\end{array}$ \\
\hline (ch) & $4^{\circ} \mathrm{C}$ & 8.6 to 65.9 ) & -1.4 & -0.3 & -2.6 & 0.85 & -1.2 & -3.2 & -7.1 & $24 \mathrm{~h}$ & not stable $p<0.0001$ & $\begin{array}{l}4.1(-1.5 \text { to } 9.7) ; \\
24 \mathrm{~h}\end{array}$ \\
\hline MO-WX & RT & 246.5 & -0.4 & -4.75 & 11.0 & 4.5 & 85.3 & 106.8 & 104.3 & $8 \mathrm{~h}$ & $\begin{array}{c}1.72(-2.3 \text { to } 5.7) ; \\
8 \mathrm{~h}\end{array}$ & $\begin{array}{l}28.4(19.9 \text { to } 36.9) ; \\
24 \mathrm{~h}\end{array}$ \\
\hline (ch) & $4^{\circ} \mathrm{C}$ & (237 to 260 ) & 6.0 & 6.0 & 8.0 & 28.0 & 42.0 & 31.0 & 62.5 & $6 \mathrm{~h}$ & $\begin{array}{c}-0.2(-4.5 \text { to } 4.0) ; \\
6 \mathrm{~h}\end{array}$ & $\begin{array}{c}\text { no clinical } \\
\text { impact over } 48 \mathrm{~h}\end{array}$ \\
\hline MO-WY & RT & 706.5 & 8.50 & -21.0 & 30.0 & 32.0 & 203.0 & 314.0 & 325.0 & $8 \mathrm{~h}$ & $\begin{array}{c}0.6(-4.6 \text { to } 5.8) ; \\
2 \mathrm{~h}\end{array}$ & $\begin{array}{c}24.9(11.6 \text { to } 38.2) ; \\
24 \mathrm{~h}\end{array}$ \\
\hline & $4^{\circ} \mathrm{C}$ & & -14.5 & -37.0 & -5.0 & -4.0 & 1.50 & 11.5 & 84.0 & $36 \mathrm{~h}$ & $\begin{array}{l}0.1(-8.0 \text { to } 8.2) ; \\
24 \mathrm{~h}\end{array}$ & $\begin{array}{l}0.1(-8.2 \text { to } 8.2) ; \\
24 h\end{array}$ \\
\hline MO-WZ & RT & & -14.3 & -13.2 & 9.45 & 38.0 & 344.5 & 636.3 & 525.3 & $8 \mathrm{~h}$ & $\begin{array}{c}5.4(-2.6 \text { to } 13.4) \\
8 \mathrm{~h}\end{array}$ & $\begin{array}{l}5.4(-2.6 \text { to } 13.4) ; \\
8 \mathrm{~h}\end{array}$ \\
\hline (ch) & $4^{\circ} \mathrm{C}$ & & 28.0 & 42.0 & 98.0 & 101.0 & 206.0 & 234.0 & 334.0 & $4 \mathrm{~h}$ & $\begin{array}{c}3.8(-2.3 \text { to } 9.8) \\
6 \mathrm{~h}\end{array}$ & $\begin{array}{c}14.3(11.2 \text { to } 17.5) \\
8 \mathrm{~h}\end{array}$ \\
\hline
\end{tabular}




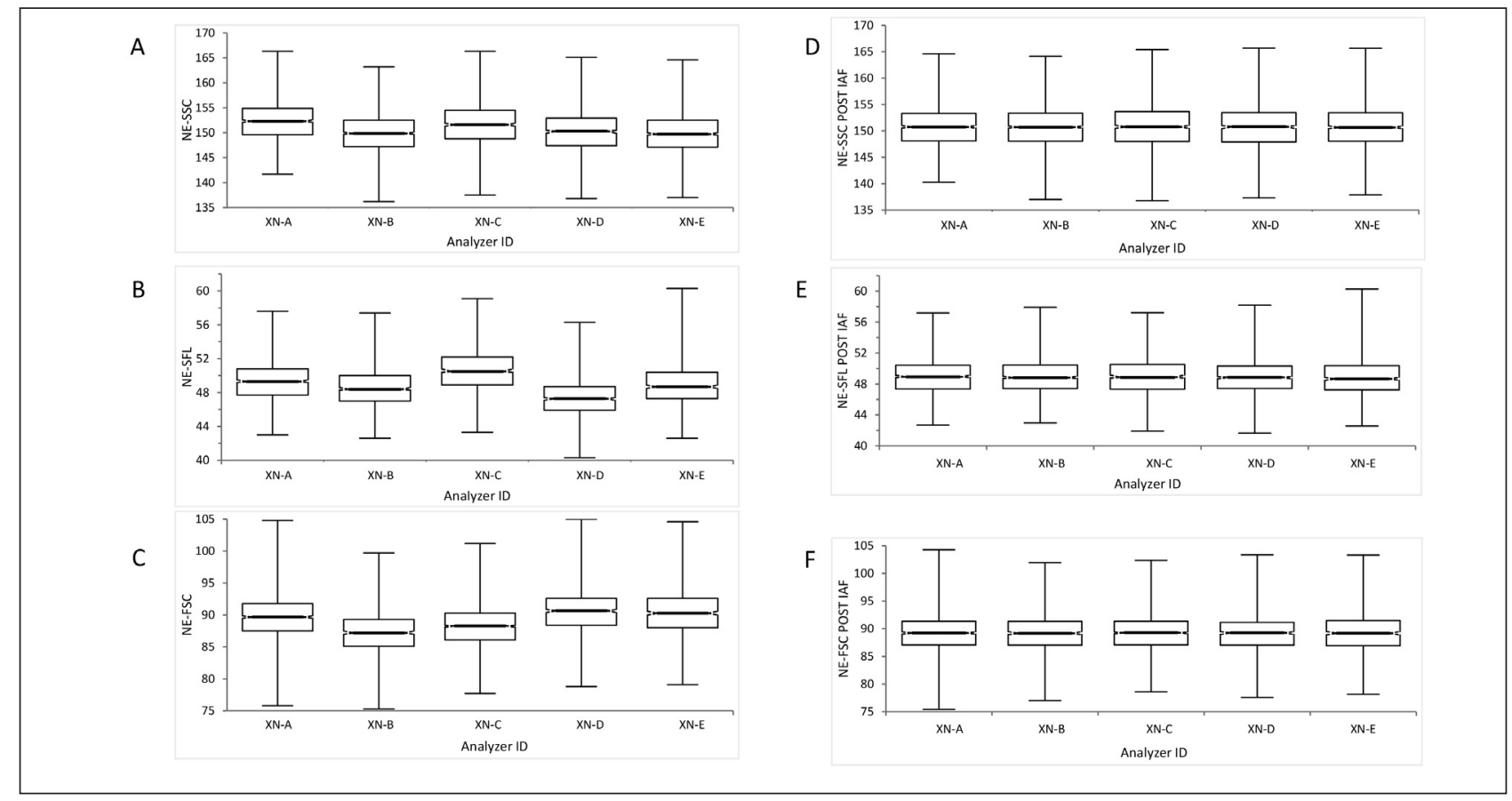

Figure 1 Neutrophils cell population data before and after application of instrumental alignment factor of November (Moving Average November 2015) on each XN module (i.e. XN-A, B, C, D, E). The box plot shows the median value as line. The 1st and $3 \mathrm{rd}$ as a box and the minimum and maximum as whiskers with the end cap for each parameter and for each $\mathrm{XN}$ module.

a) Median value NE-SSC before application of instrumental alignment factor; b) median value NE-SFL before application of instrumental alignment factor; c) median value NE-FCS before application of instrumental alignment factor; d) median value NE-SSC after application of instrumental alignment factor; e) median value NE-SFL after application of instrumental alignment factor; $f$ ) median value NE-FCS after application of instrumental alignment factor.

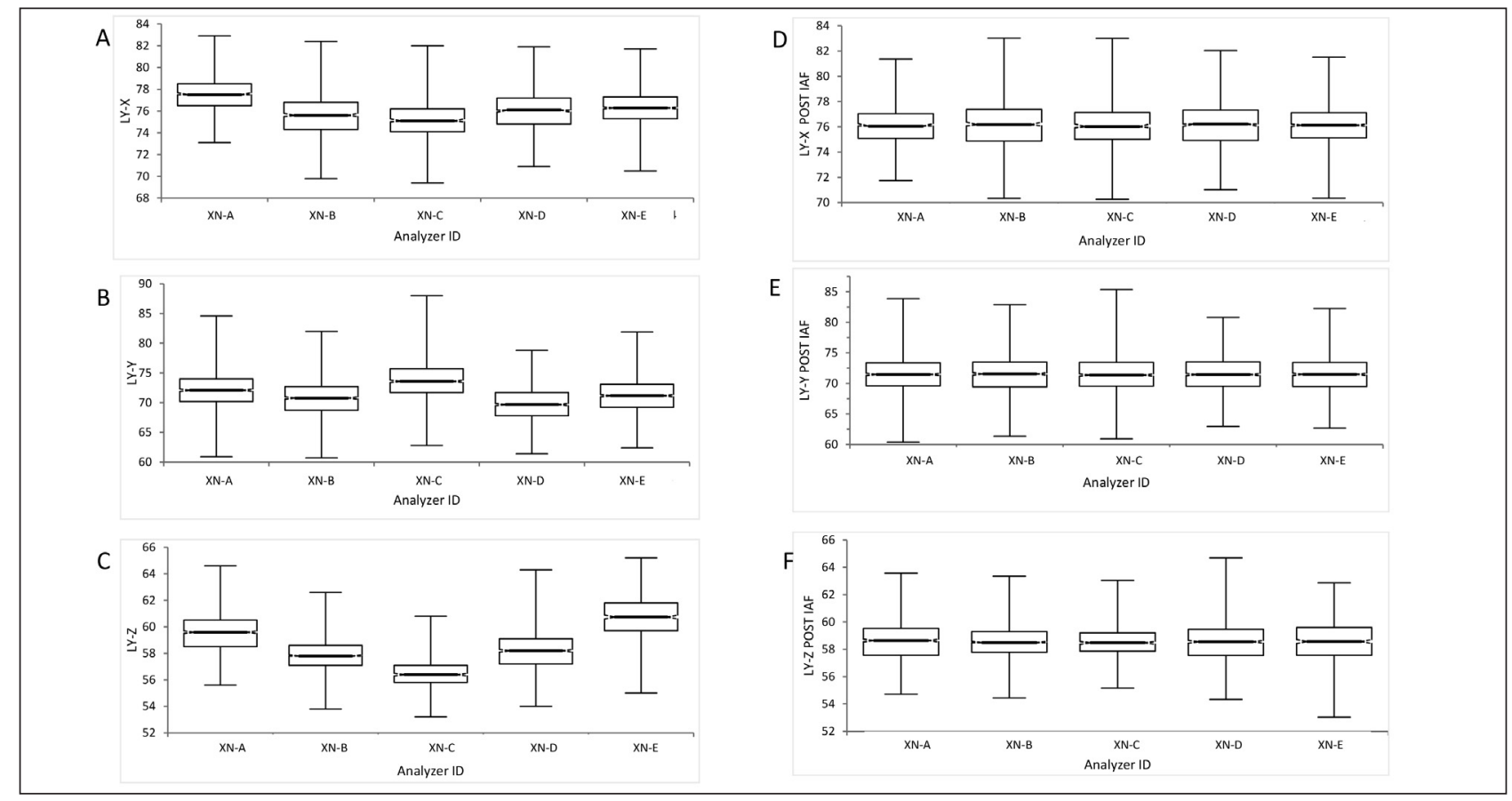

Figure 2 Lymphocytes cell population data before and after application of instrumental alignment factor of November (Moving Average November 2015) on each XN module (i.e. XN-A, B, C, D, E). The box plot shows the median value as line. The 1st and 3rd as a box and the minimum and maximum as whiskers with the end cap for each parameter and for each $\mathrm{XN}$ module.

a) Median value LY-X before application of instrumental alignment factor; $b$ ) median value LY-Y before application of instrumental alignment factor; c) median value LY-Z before application of instrumental alignment factor; d) median value LY-X after application of instrumental alignment factor; e) median value LY-Y after application of instrumental alignment factor; f) median value LY-Z after application of instrumental alignment factor. 


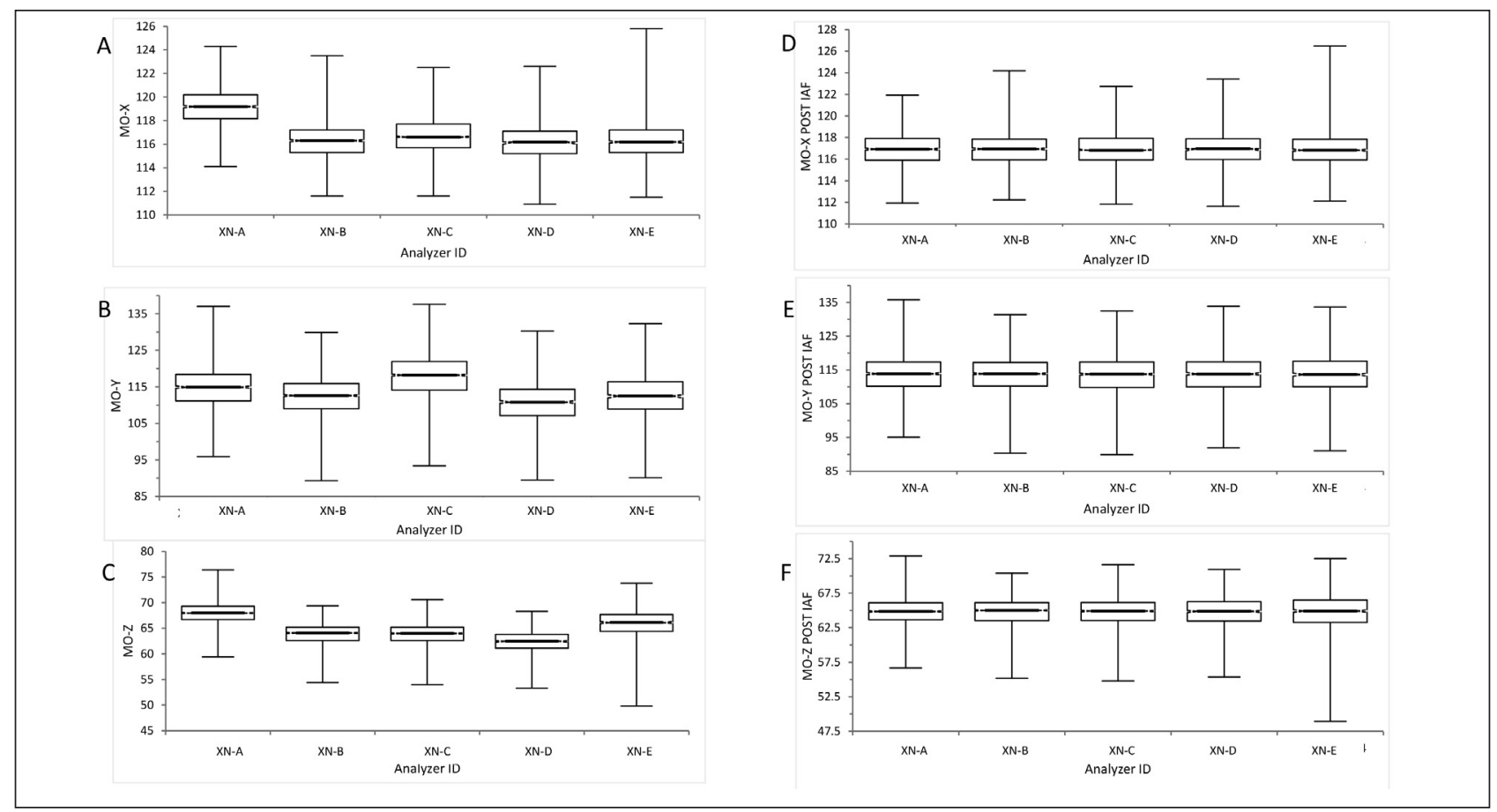

Figure 3 Monocytes cell population data before and after application of instrumental alignment factor of November (Moving Average November 2015) on each XN module (i.e. XN-A, B, C, D, E). The box plot shown the median value as line. The 1 st and $3 \mathrm{rd}$ as a box and the minimum and maximum as whiskers with the end cap for each parameter and for each $\mathrm{XN}$ module.

a) Median value MO-X before application of instrumental alignment factor; b) median value MO-Y before application of instrumental alignment factor; c) median value MO-Z before application of instrumental alignment factor; d) median value $\mathrm{MO}-\mathrm{X}$ after application of instrumental alignment factor; e) median value MO-Y after application of instrumental alignment factor; f) median value MO-Z after application of instrumental alignment factor.

\section{Discussion}

The aim of the study was to assess the current degree of CPD harmonization, using five separate Sysmex $\mathrm{XN}$-modules allocated to the same clinical laboratory. Regarding the imprecision of the separate modules, only NE-SFC and LY-Y fulfilled the established specifications for desirable imprecision (14). Other CPD parameters only met the minimum acceptable level of imprecision, whilst those reflecting cellular dispersion (i.e., NE, LY and MO-WX, WY and WZ) displayed unacceptable imprecision. This important finding should hence persuade the manufacturer to plan additional efforts for improving the current analytical performance.

The study design for verification of instrumental alignment was critical. Our study included five XNmodules, but specific control material with target values is not available. For this reason, we decided to use the OMV obtained for each parameter as the reference value. Our results confirm that comparing the Bias\% of each $\mathrm{XN}$-Module to $\mathrm{B}_{\mathrm{APS}} \%$ may lead to incorrect assumptions, thus failing to detect a lack of instrumentation alignment. Conversely, the use of $\mathrm{B}_{\mathrm{AGL}} \%$ seems much more appropriate for identifying poor instrumental alignment, as predictable. Therefore, data generated after the application of IAF correction attests that this harmonization approach may more effectively improve the alignment of CPD parameters among different $\mathrm{XN}$ modules.

According to our data, the performance of CPD parameters seems stable over time and, even more importantly, we could demonstrate that the use of data generated from normal routine samples (i.e., the MA population) may be reliably used as an inexpensive approach to improve harmonization of CPD parameters among separate $\mathrm{XN}$-modules allocated in the same laboratory. The approach of calculating IAF is not new to the field of laboratory medicine, since it is successfully used for improving the harmonization of coagulation testing among separate analyzers using the identical reagents (i.e., adopting the socalled instrument specific international sensitivity index; ISI) (23). Notably, once the XN-Check is certified for monitoring the analytical performance of CPD parameters, this control material may then be used for daily internal quality control assessment, for eventually highlighting the need for recalibration and thus ultimately improving inter-laboratory comparability.

Regarding the stability of whole blood samples at different temperatures, the data obtained in this study are quite comparable, and all CPD parameters seem more stable when maintained at room temperature for up to 2-4 hours. Interestingly, samples were found to be more stable at room temperature than at 
$4^{\circ} \mathrm{C}$, and this is probably due to the unfavorable effect of low temperatures on leukocyte physiology (24). When the $\mathrm{B} \%$ from results obtained immediately after collection (TO) was compared to the RCV, results were seemingly acceptable for at least 8 hours for all the CPD parameters, regardless of storage temperature. This is probably satisfactory, especially for samples collected from peripheral phlebotomy facilities which are then shipped for being analyzed in central or reference laboratories.

\section{Conclusions}

In conclusion, we found acceptable imprecision of all CPD parameters, except for those reflecting the dispersion of cellular clusters. Therefore, it seems now advisable to use only those parameters meeting the minimum analytical quality requirements in routine clinical practice, whilst the other parameters still need improvement of analytical performance before being widely used. Due to the lack of reference quality control materials, we also showed that the use of

\section{References}

1. Lecompte TP, Bernimoulin MP. Novel Parameters in Blood Cell Counters. Clin Lab Med 2015; 35: 209-24.

2. Briggs C, Longair I, Kumar P, Singh D, Machin SJ. Performance evaluation of the Sysmex haematology $\mathrm{XN}$ modular system. J Clin Pathol 2012; 65: 1024-30.

3. Buttarello M. Quality specification in hematology: the automated blood cell count. Clin Chim Acta 2004; 346: 45-54.

4. Le Roux G, Vlad A, Eclache V, Malanquin C, Collon JF, Gantier $M$, et al. Routine diagnostic procedures of myelodysplastic syndromes: value of a structural blood cell parameter (NEUT-X) determined by the Sysmex XE2100TM. Int J Lab Hematol 2010; 32: 237-43.

5. Park SH, Kim HH, Kim IS, Yi J, Chang CL, Lee EY. Cell population data NE-SFL and MO-WX from Sysmex XN3000 can provide additional information for exclusion of acute promyelocytic leukemia from other acute myeloid leukemias: a preliminary study. Ann Lab Med 2016; 36: 7-10.

6. Furundarena JR, Araiz M, Uranga M, Sainz MR, Agirre A, Trassorras M, et al. The utility of the Sysmex XE-2100 analyzer's NEUT-X and NEUT-Y parameters for detecting neutrophil dysplasia in myelodysplastic syndromes. Int J Lab Hematol 2010; 32: 360-6.

7. Park SH, Park CJ, Lee BR, Nam KS, Kim MJ, Han MY, et al. Sepsis affects most routine and cell population data (CPD) obtained using the Sysmex XN-2000 blood cell analyzer: neutrophil-related CPD NE-SFL and NE-WY provide useful information for detecting sepsis. Int J Lab Hematol 2015; 37: 190-8. results generated on a large number of normal routine samples (i.e., the MA population) may be a reliable approach for checking between-instrument harmonization of CPD-parameters, by calculation of instrument-specific IAF. Nevertheless, the availability of both calibration and quality control materials for these parameters is highly advisable in the future, since traceable standards may allow further improvements in the process of widespread harmonization of CPD parameters. Finally we showed that whole blood samples may be stable for up to 2-4 hours for analysis of the vast majority of CPD parameters.

Disclosures: Dr. Ramon Simon-Lopez Sysmex Corporation Consultancy, honoraria.

Acknowledgments: We are thankful to Dr. Aurelio Pacioni for technical support.

\section{Conflict of interest statement}

The authors state that they have no conflicts of interest regarding the publication of this article.

8. Buoro S, Seghezzi M, Vavassori M, Dominoni P, Apassiti Esposito $S$, et al. Clinical significance of cell population data (CPD) on Sysmex XN-9000 in septic patients withour without liver impairment. Ann Transl Med 2016; 4: 418-22.

9. Lou Y, Lin J, Chen H, Zhang J, Peng S, Kuang M. Utility of neut- $X$, neut- $Y$ and neut- $Z$ parameters for rapidly assessing sepsis in tumor patients. Clin Chim Acta 2013; 422: 5-9.

10. Brisou G, Manzoni D, Dalle S, Felman P, Morel D, Boubaya $M$, et al. Alarms and parameters generated by hematology analyzer: new tools to predict and quantify circulating Sezary cells. J Clin Lab Anal 2015; 29: 15361.

11. Furundarena JR, Uranga A, Sainz MR, González C, Uresandi N, Argoitia N, et al. Usefulness of the lymphocyte positional parameters in the Sysmex XN haematology analyser in lymphoproliferative disorders and mononucleosis syndrome. Int J Lab Hematol 2018; 40: $41-8$.

12. Plebani M, Lippi G. Is laboratory medicine a dying profession? Blessed are those who have not seen and yet have believed. Clin Biochem 2010; 43: 939-41.

13. Clinical Laboratory Standards Institute. Evaluation of Precision of Quantitative Measurement Procedures; approved guideline - third edition. CLSI document EP05-A3E. Clinical Laboratory Standards Institute: Wayne, PA; 2014.

14. Buoro S, Carobene A, Seghezzi M, Manenti B, Pacioni A, Ceriotti $F$, et al. Short- and medium-term biological variation estimates of leukocytes extended to differential 
count and morphology-structural parameters (cell population data) in blood samples obtained from healthy people. Clin Chim Acta 2017; 473: 147-56.

15. Fraser CG. Biological Variation: From Principles to Practice. Washington, DC, USA: AACC Press; 2001.

16. Petersen PH, Fraser CG, Westgard JO, Larsen ML. Analytical goal setting for monitoring patients when two analytical methods are used. Clin Chem 1992; 38: 2256-60.

17. Calleja J. Parallel Processing and Maintaining Adequate Alignment between Instruments and Methods. Clin Biochem 2008; 29: 71-6.

18. Ghasemi A, Zahedias S. Normality Tests for Statistical Analysis: A Guide for Non-Statisticians. Int J Endocrinol Metab 2012; 10: 486-9.

19. Shapiro SS, Wilk MB. An analysis of variance test for normality (complete samples). Biometrika 1965; 52: 591-611.
20. Spurier D. Additional Tables for Steel-Dwass-CritchlowFligner Distribution-Free Multiple Comparisons of Three Treatments. Communications in Statistics - Simulation and Computation 2007; 35: 441-6.

21. Jørgensen EP. Leadership and management in clinical biochemistry. J Med Biochem 2017; 36: 216-9.

22. Arslan DF, Karakoyun I, Basok IB, Aksit ZM, Celik E, Dogan K, Duman C. The effects of education and training given to phlebotomists for reducing preanalytical errors. J Med Biochem 2018; 37: 172-80.

23. Brien WF, Crawford L, Raby A, Richardson H. In-house calibration of the international sensitivity index or calibration curve for determination of the international normalized ratio. Arch Pathol Lab Med 2004; 128: 308-12.

24. Aderibigbe OM, Priel DL, Lee CC, Ombrello MJ, Prajapati $\mathrm{VH}$, Liang MG, et al. Distinct Cutaneous Manifestations and Cold-Induced Leukocyte Activation Associated With PLCG2 Mutations. JAMA Dermatol 2015; 151: 627-34.

Received: January 22, 2018

Acepted: February 21, 2018 